\title{
Development of Segmented Semiconductor Arrays for
}

\section{Quantum Imaging}

\author{
B. Mikulec, \\ Medipix2 Collaboration \\ CERN, ETT division, $\mathrm{CH}-1211$ Geneva 23, Switzerland; \\ now with the University of Geneva, 30, quai Ernest-Ansermet, $\mathrm{CH}-1211$ Geneva 4, \\ email: bettina.mikulec@cern.ch
}

PACS: 29.40.Gx, 29.40.Wk, 87.59

Keywords: quantum imaging, photon counting, semiconductor detectors, flat field correction, X-ray imaging

\begin{abstract}
The field of pixel detectors has grown strongly in recent years through progress in CMOS technology, which permits many hundreds of transistors to be implemented in a square area with a side of 50$200 \mu \mathrm{m}$. Pulse processing electronics with noise of the order of $100 \mathrm{e}^{-} \mathrm{rms}$ permits to distinguish photons of a few keV from background noise. Techniques are under development, which should allow single chip systems (area $\sim 1 \mathrm{~cm}^{2}$ ) to be extended to larger areas.

This paper gives an introduction into the concept of quantum imaging using direct conversion in segmented semiconductor arrays. An overview of projects from this domain using strip, pad and in particular hybrid pixel detectors will be presented. One of these projects, the Medipix project, is described in more detail. The effect of different correction methods like threshold adjustment and flat field correction is illustrated and new measurement results and images presented.
\end{abstract}

\section{Introduction}

For more than a century film has dominated both visible and X-ray imaging as detection medium. Nowadays, microelectronics opens new ways for the conception of modern imaging detectors. Miniaturisation allows the readout electronics to be attached directly to tiny sensor channels that provide good spatial resolution and low noise, and in particular to process individual incoming photons 'on the fly' prior to storage and image processing.

In general, the sensor medium has to be chosen such that it produces the highest possible signal for each particle to be detected and that the signal is uniform all over the detection area. Besides, the 
determined position should correspond to the real impact point of the impinging particle. This is best achieved by direct conversion in thin detectors with as few conversion stages as possible. High-Z, high resistivity semiconductor materials are very good candidates, as they combine the advantages of high stopping power (detector thickness $<500 \mu \mathrm{m}$ possible), large signal (about an order of magnitude more primary charge carriers than in gaseous detectors) and excellent spatial resolution (defined by the planar process [1],[2]]. Silicon is certainly the best known and most frequently used sensor material with very good homogeneity (although studies will be presented later in this paper showing microscopic nonuniformities occurring also in silicon sensors), but due to its low atomic number its attenuation properties for X-rays are only mediocre. A $300 \mu \mathrm{m}$ thick silicon detector converts only $\sim 26.7 \%$ of $20 \mathrm{keV} \mathrm{X}$-rays and $\sim 2 \%$ of $60 \mathrm{keV} \mathrm{X}$-rays [3]. Therefore a lot of effort has been put into the development of new compound semiconductor sensors like semi-insulating (SI) GaAs [4][12], epitaxial GaAs [5] [13], 20], CdTe and CZT $\left(\mathrm{Cd}_{1-\mathrm{x}} \mathrm{Zn} \mathrm{n}_{\mathrm{x}} \mathrm{Te}\right)$ [5] [6], 21] [30], or more exotic compounds like thallium bromide (TIBr), indium phosphide $(\mathrm{InP})$, mercuric iodide $\left(\mathrm{Hgl}_{2}\right)$ or lead iodide $\left(\mathrm{Pbl}_{2}\right)$ [5] [6], [31] 39]. These materials are superior in terms of photon attenuation, but there are still many problems to be solved concerning charge trapping, impurity concentration, polarisation, contact quality and non-uniformities in terms of charge collection efficiency as well as unstable leakage currents over the sensor area. As a consequence, the attached readout electronics have to cope with these new requirements. Silicon sensors are usually $p$ on $\mathrm{n}$ material, and therefore the electronics channels were designed mostly for hole collection. This has to be changed if one wants to use e.g. CZT sensors where a big fraction of the holes gets trapped before collection. It is the reason why most of the new readout chips will be designed to be sensitive to negative input signals as well as positive ones as will be mentioned later. Individual leakage current compensation per readout channel can also be an important feature to correct for variable leakage currents [40] in order to improve signal-to-noise uniformity. Moreover, new upcoming applications ask for specific signal processing which all results in a big challenge for the ASIC designers.

Especially in the case of pixel detectors where the pixel size defines the available space for the pixel readout electronics the implementation of all the additional functionality requires to follow the technology advances. G.E. Moore predicted the amazing progress in microelectronics already in 1965 [41] The socalled Moore's law refers to his observation that in microelectronics the density of electronic components increases exponentially with time [43][44] Progress on the electronics side therefore goes much faster than on the sensor side.

\footnotetext{
${ }^{1}$ He reviewed his prediction in 1975 saying that the number of transistors per chip would double every 18 months instead of every year [42].
} 


\section{Quantum imaging with hybrid semiconductor detectors}

Quantum imaging was only made possible through these advances in microelectronics. It needs a clean discrimination between signal and noise that could only be achieved with the availability of low-noise, pulse-processing front-end electronics, connected to small sensor cells of low capacitance. For quantum imaging, the readout architecture has to fulfil two requirements:

i. A signal gets attributed to a particle and subsequently processed only when it exceeds a threshold that is set comfortably far away from the noise level.

ii. In the case of small pixel dimensions the charge cloud may be distributed over several detector channels, but in the end each converted particle or photon has to be assigned to exactly one image element.

Photon counting is the simplest example for quantum imaging, where each signal passing the threshold is counted up, but there are many more ways to do quantum imaging by actively processing the overthreshold signal. One could imagine for example to determine the signal height that is proportional to the deposited energy and subsequently store it in a corresponding ADC channel. This opens the possibility to give offline a weight factor to the content of each ADC channel. The ideal weight factor to achieve maximum detective quantum efficiency (DQE) using spectral X-ray sources is proportional to $E^{-3}(E \ldots$ incident photon energy) [45],446], which reflects the attenuation properties of the objects to be imaged. Charge integrating devices like film or $\mathrm{CCDs}^{2}$ result in a weight factor proportional to the deposited energy. Therefore they are already one order of magnitude worse than photon counting devices, which give each signal an equal weight of 1 independent from their energy deposition.

The main advantages of quantum imaging are the following:

- Suppression of low-frequency noise (including e.g. dark current) can effectively be performed with pulse-processing front-end electronics. This increases the signal-to-noise ratio (SNR) of the image. As opposed to charge integrating systems low rate imaging can be performed, even up to acquisition times of several hours (see e.g. [47]).

- Counting systems exhibit a perfectly linear behaviour over their entire dynamic range [12][48] [49] The dynamic range can be chosen arbitrarily according to the application in view. It is only limited by space considerations of the electronics channel (especially in the case of pixel detectors).

\footnotetext{
${ }^{2}$ CCD... charge coupled device
} 
- The setting of a threshold makes it possible to discriminate not only noise from signal, but also lower from higher energies. Strongly Compton scattered events can therefore be suppressed with monoenergetic sources reducing image blurring. Synchrotron experiments like X-ray crystallography can make use of this feature as well to remove the X-ray fluorescence background [50].

- Multiple thresholds can be implemented as well. This opens completely new perspectives for imaging with spectral sources. Two thresholds allow for example to create subtraction images (e.g. K-edge subtraction) online with one shot (instead of two takes and offline subtraction, halving already the patient dose). Several thresholds can be combined with a multi-bit ADC per channel to produce a sort of 'colour' X-ray image. Moreover, energy weighting can be applied for contrast enhancement or various other kinds of analysis.

This paper focuses on quantum imaging with direct conversion segmented semiconductor arrays. The segmentation of a semiconductor sensor is realised either in a 1-dimensional way (strip detectors) or 2dimensional (pixel detectors or pad detectors when the pixel size is of the order of $\mathrm{mm}$ or larger) where the spatial resolution is mainly defined by the electrode pitch. The requirement of high quantum efficiencies in X-ray imaging applications together with the desired direct conversion of the X-rays into electric charge leads to the usage of the hybrid detector concept. Hybrid detectors consist of a segmented semiconductor sensor chip and a separate CMOS readout chip where each sensor channel is electrically connected to one electronics channel via so-called bump bonds (usually either made of solder, indium or gold) in the case of pixel detectors or wire bonds for strip and pad detectors. This concept allows for an independent optimisation of both the sensor chip and the electronics chip in terms of performance, thickness of the sensor etc. as well as the use of various semiconductor sensor materials.

In the following section of this paper a selection of various quantum imaging projects using segmented semiconductor detectors is presented. The Medipix project will be discussed in more detail as one example of such a quantum imaging project.

\section{State of the art}

For strip and pad detectors it is possible to use conventional large components for the digital logic and an ASIC is required only for the analogue signal processing. To perform quantum imaging with hybrid semiconductor pixel detectors, the entire amplification, signal shaping, discrimination and digital logic chain has to fit into a tiny area commensurate with the pitch of the sensor pixels (which is typically in the order $50-200 \mu \mathrm{m})$. 
In the description of the projects mentioned below an attempt is made to highlight particularities of the electronics designs, the geometry, the mechanical arrangement or an interesting underlying concept. While there is no attempt to provide a comprehensive list of all existing projects, a large number of pixel projects are reported. The applications that drive the system design are also considered.

\subsection{Projects based on strip detectors}

\subsubsection{SYRMEP 3}

The aim of the SYRMEP project is the development of a detector for digital diagnostic radiology with synchrotron radiation at the third generation light source ELETTRA, Trieste, Italy [51]. The beam energy for diagnostic radiology of soft tissue (in particular for mammography) lies in the range of $15-30 \mathrm{keV}$, for which their detector has to show highest possible detection efficiency to achieve low patient dose. The SYRMEP project adopted therefore a geometry called 'edge-on', where the incoming X-rays impinge into the side edge of a silicon microstrip sensor, parallel to the strips (see fig. 1). This increases the effective absorption length from a few hundred $\mu \mathrm{m}$ to about $1 \mathrm{~cm}$ (=strip length). The microstrip detector acts in this configuration as a pixel detector, where the pixel size is defined by the strip pitch in one direction $(200 \mu \mathrm{m})$ and by the wafer thickness in the other one $(300 \mu \mathrm{m})$. There is an undepleted part of the silicon sensor at the entrance window of the X-rays given approximately by the distance between the scribeline and the $p+$ implants of the strips, which results in a dead layer. This dead layer was minimised in their sensor design yielding a detection efficiency $>80 \%$ for $20 \mathrm{keV}$ X-rays [51] [53]. The 256 strips of the sensor are read out by 8 ASICs designed in AMS $1.2 \mu \mathrm{m}$ technology with the name Castor ${ }^{1}$, where each channel consists basically of a low-noise charge sensitive preamplifier, shaping amplifier, discriminator and a 16-bit counter [54] [55]. To increase the sensitive area to $\sim 50 \times 1 \mathrm{~mm}^{2}$ a stack of 3 detection layers has been built [51] Phantom images at reduced dose were obtained with this system scanning the object and using sampling steps smaller than the pixel size [51], One limitation of this system is the low counting rate capability of the Castor chip yielding a maximum counting rate of $\sim 10 \mathrm{kHz} /$ channel and therefore unacceptably long examination times of a few minutes [51] (breathing artefacts of the patient). The efforts of the SYRMEP/FRONTRAD project consist now in decreasing further the dead layer and especially in designing a new version of the ASIC called FROST [56].

\footnotetext{
${ }^{3}$ SYnchrotron Radiation for MEdical Physics

${ }^{4}$ Counting and Amplifying SysTem fOr Radiation detection
} 


\subsubsection{The quantum $X$-ray radiology apparatus}

A quantum X-ray apparatus was developed aiming specifically at radiological spinal column examinations [57][58]. This project also adopted the silicon edge-on microstrip principle described above. During the examination the patient is standing and the X-ray generator together with a collimation system (to define the beam and to avoid scattered radiation) and the box containing the detector is scanned vertically over a distance of up to $1.2 \mathrm{~m}$. A silicon sensor thickness of $500 \mu \mathrm{m}$ and a strip pitch of $500 \mu \mathrm{m}$ were defined for this application. Eight detectors are arranged in a linear array covering a length of about $50 \mathrm{~cm}$ with a dead zone of 3 strips in-between the detectors. Due to the higher beam energy the microstrips were chosen to have a length of $\sim 5 \mathrm{~cm}$ to yield a stopping power $>90 \%$ for $50 \mathrm{keV} \mathrm{X}$-rays. Moreover, the strip geometry was adapted to avoid any parallax. Signal processing is performed by an analogue followed by a digital ASIC [59]. The analogue ASIC is sensitive to both negative and positive signal input; each channel comprises individual leakage current compensation, a charge sensitive preamplifier with two different gains, a shaper with variable peaking time and a high-speed output buffer with a gain of 10 . Moreover, an automatic output voltage offset correction is performed to avoid channel-to-channel dispersions. Each channel of the digital ASIC includes a window discriminator with adjustable thresholds, a 16-bit counter with overflow bit and a $16+1$ bit buffer. The system can continuously acquire data and is linear up to $200 \mathrm{keV}$. A new version of the detection system is being designed to improve the spatial resolution, eliminate the dead areas between detectors and introduce a spectroscopic analysis of the incident radiation [57].

\subsubsection{Mamea Imaging $A B^{\square}$}

This Swedish company produces detection systems based on silicon microstrip detectors, which are integrated into a mammography system called Sectra MicroDose Mammography ${ }^{\mathrm{TM}}$ and commercialised by Sectra Imtec $\mathrm{AB}^{\mathrm{E}}$. The main difference to the previous two projects consists of the 'almost edge-on' geometry where the silicon sensor is tilted by a small angle around $4^{\circ}$ and illuminated either through the front- or the back-side [46] This reduces the dead entrance layer and allows even using a multiguardring structure. Quantum efficiencies of $90 \%$ for a filtered $30-k V p$ tungsten spectrum have been reported with the $500 \mu \mathrm{m}$ thick sensors. Each of the sensors in the detector stack comprises 768 strips with a pitch of $50 \mu \mathrm{m}$. A slight fanout of the strips compensates for the beam divergence. Signal processing is performed by a 128-channel readout ASIC. Each channel comprises preamplifier, shaper,

\footnotetext{
${ }^{5}$ http://www.mamea.com/

${ }^{6}$ Sectra Imtec AB, Teknikringen 2, SE - 58330 Linköping, Sweden; http://www.sectra.se/medical/
} 
a discriminator with 3-bit threshold adjustment and a counter. Maximum counting rates $>1 \mathrm{MHz} / \mathrm{channel}$ can be achieved and yield an acquisition time between 5-7 s depending on the thickness and/or density of the examined breast [60]. A prototype ASIC has been made with a coincidence circuit to take care of charge sharing effects between adjacent strips. An evaluation of this chip is underway.

\subsubsection{Diffex}

Diffex is a recently designed linear solid-state module mainly aiming at $\mathrm{X}$-ray powder diffraction in synchrotron beams [61]. In powder diffraction experiments the sample is illuminated with monochromatic $\mathrm{X}$-rays in the energy range $5-20 \mathrm{keV}$, and the atoms in the powder produce diffraction cones. The measurement of the cone apertures is used to determine the atomic composition of the sample. Due to the symmetry it is sufficient to use an adequately long linear array, which samples the rings along the radial axis (see fig. 2 [62]]. A silicon microstrip detector has been designed, which consists of two columns of 512 sensor elements on a pitch of $100 \mu \mathrm{m}$, each $500 \mu \mathrm{m}$ long. The columns are staggered by $50 \mu \mathrm{m}$ with respect to one another. Sensors of 300 and $500 \mu \mathrm{m}$ thickness have been produced. 16 DFX ASICs read out the module. The DFX chip was designed in a $0.25 \mu \mathrm{m}$ CMOS technology. Each channel comprises a low-noise charge sensitive preamplifier, a shaper, two comparators, a 4-bit ADC and eighteen 15-bit counters with shadow registers for parallel, virtually dead-time free readout. Users can choose between four different signal processing modes: normal counting mode; high counting rate mode in excess of $1 \mathrm{MHz}$; in the energy resolution mode the time-over-threshold is used and each channel works effectively as a 4-bit Multi-channel Analyser; the last mode works like the previous one, but includes a charge sharing check with the help of the second comparator and two special counters. The DFX is currently under test.

\subsection{Projects based on pixel detectors}

\subsection{1 $\quad X P A D^{\square}$}

Third generation synchrotrons like ELETTRA or ESRF in Grenoble, France, feature extremely high photon fluxes. A pixel detector is under development aimed at X-ray crystallography with the final goal to cover an area of $25 \times 25 \mathrm{~cm}^{2}$, a count rate capability around $10^{7}$ photons/s/pixel, dynamic range $>10^{9}$ and fast frame rate [63] The prototype module is composed of ten XPAD readout chips and makes use of $300 \mu \mathrm{m}$ thick silicon sensors, which were developed for the Delphi experiment at CERN. This yields an

\footnotetext{
${ }^{7}$ X-ray Pixel chip with Adaptable Dynamics
} 
active area of $4 \times 1.6 \mathrm{~cm}^{2}$ (see fig. 3 [64]]. The space between two adjacent chips is not dead as two sensor pixels are combined to one 'superpixel', which is read out by one electronics channel. XPAD was designed in AMS $0.8 \mu \mathrm{m}$ technology, is arranged in 24 columns of 25 square pixels measuring $330 \times 330 \mu \mathrm{m}^{2}$ and is sensitive to positive and negative signal input. Besides the 4 bits available for threshold adjustment and a counting rate/pixel in excess of $1 \mathrm{MHz}$ the main characteristics of the ASIC is its large dynamic range. There is a 16-bit counter in each channel and another 16-bit counter per channel in an external memory board. The readout system scans the overflow of the pixel counters at $33 \mathrm{MHz}$ and adds the overflows to the corresponding counters in the memory board. This takes about $3 \mathrm{~ms}$ for the entire prototype module, which is shorter than the time it takes to fill up the pixel counters. This architecture results in an effective dynamic range of 32 bits. In fig. 4 [64] showing an X-ray diffraction image this dynamic range can be appreciated. However, many black spots are visible in the image. They were disabled as their threshold was out of range. To correct this problem a new chip called XPAD-2 was designed which was successfully tested [65] Bump-bonding of XPAD-2 to CdTe and Si sensors is underway and modules of $6 \times 6 \mathrm{~cm}^{2}$ in a 'roof tile' architecture are planned. Moreover, a new ASIC, XPAD-3, is under design in a $0.25 \mu \mathrm{m}$ technology to increase functionality and decrease the pixel size to $150 \times 150 \mu \mathrm{m}^{2}$.

\subsubsection{PILATUS ${ }^{\mathrm{B}}$}

The PILATUS project, located at the Swiss Light Source (SLS), has aims similar to those of XPAD. Their most recent ASIC is called SLS06 and was designed in DMILL $0.8 \mu \mathrm{m}$ technology (radiation hard) [66], It is arranged in a matrix of 44 columns and 78 rows of square pixels measuring $217 \mu \mathrm{m}$ on the side. The threshold can be tuned with 4 bits and the pixel counters have a depth of 15 bits. xy-addressing was implemented instead of a shift register for readout. One of the advantages of this architecture is a better tolerance for defects. Analogue signal outputs are available for testing purposes. One PILATUS silicon module consists of $2 \times 8$ SLS06 chips and covers an area of $\sim 8 \times 3.5 \mathrm{~cm}^{2}$ (with 'superpixels' at chip boundaries) [67] Bump-bonding (In bumps) is done in-house. The group has built the largest contiguous area pixel detector plane up to now with three PILATUS modules in a row (see fig. 5). This detector fulfils already the requirement of area coverage in one direction $(24 \mathrm{~cm}$; gap of $2.4 \mathrm{~mm}$ between the modules); the $\sim 165000$ pixels can be read out in $5 \mathrm{~ms}$. The design of a future ASIC in $0.25 \mu \mathrm{m}$ technology is

\footnotetext{
${ }^{8}$ PIxeL ApparaTUs for the SLS
} 
planned with the main goal to increase the counter depth to 18 bits and at the same time decrease the pixel size below $200 \mu \mathrm{m}$.

\subsubsection{LAD1 ${ }^{\text {G }}$}

The aim of this project is to develop a large area photon counting detector for time resolved X-ray diffraction studies. One solution which was adopted to achieve large active areas is to tile several detector modules. A 'roof tile' architecture was chosen (see fig. 6 [62]] and should permit a $30 \times 30 \mathrm{~cm}^{2}$ area coverage with minimum dead space [68], A 64 × 64 pixel readout chip called Aladin ${ }^{10}$ was designed for the project in $0.5 \mu \mathrm{m}$ Mietec technology. Each pixel contains a discriminator with 3-bit threshold tuning and a 15-bit counter and is expected to count up to a frequency of $1 \mathrm{MHz}$. The silicon sensor pixels are square with a pitch of $150 \mu \mathrm{m}$, but the pixels of the readout chip are slightly smaller in one direction $(144 \mu \mathrm{m})$. This architecture together with special metal routing on the sensor side results in the advantage that each sensor pixel is connected to exactly one readout pixel, even in the region between readout chips (no 'superpixels') [69].

\subsubsection{MPEC}

The MPEC 2.3 is a pixel readout chip designed at the University of Bonn, Germany in AMS $0.8 \mu \mathrm{m}$ technology. The pixel array consists of a matrix of $32 \times 32$ square pixels of $200 \mu \mathrm{m}$ on the side. The chip is optimised for positive charge input, but it works also to some extent for electron collection [70] Each MPEC 2.3 pixel (as for the predecessor chip MPEC 2.1) contains two discriminators with corresponding 18-bit counters [71]. The discriminators are tuneable by storing an analogue correction voltage on a capacitor in each pixel (needs refreshing in the case of long acquisition times). The MPEC 2.1 pixel ASIC is the first CMOS pixel chip with an energy window implemented in each pixel (see fig. 7 [72]]. MPEC 2.3 has been bump-bonded to CdTe sensors, and bump-bonding to Si sensors is underway. The group developed a small and mobile 'plug \& play' system based on a laptop and USB readout for $2 \times 2$ pixel arrays 70]. A new chip design in $0.25 \mu \mathrm{m}$ technology is planned as well.

\subsubsection{DIX}

The University of Uppsala, Norway, and Ideas ASA ${ }^{13}$, Norway have developed jointly a pixel readout ASIC called ANGIE [73]. The pixel detector aims primarily at dynamic medical X-ray imaging, e.g. for

\footnotetext{
${ }^{9}$ Large Area Detector

${ }^{10}$ A Large Area Detector with Incrementors

${ }^{11}$ Multi Picture Element Counters

${ }^{12}$ DIgital X-ray Imaging
} 
angiography. Design technology is again AMS $0.8 \mu \mathrm{m}$; the pixel matrix consists of 31 columns, each with 32 pixels of $270 \times 270 \mu \mathrm{m}^{2}$ area [74]. The pixel cells are sensitive to positive and negative charge input; each cell has an externally adjustable discriminator and two analogue counters. The depth of the counters is variable up to 15 bits. It is possible to quickly switch from one counter to the other one before readout and to change at the same time the discriminator level. This allows for example offline image subtraction of two images with different energy content. Readout is performed serially at a maximum frame rate of 100 frames/s. It is planned for the near future to bump-bond a linear array of eight ASICs to a silicon sensor.

\subsubsection{DPAD}

Protein crystallography is the field of application foreseen for the DPAD pixel detector [75] High dynamic range and continuous readout are therefore essential. With this in mind, an ASIC was designed in HP $0.5 \mu \mathrm{m}$ technology. The particularity of the $16 \times 16$ square pixel array (pixel pitch $150 \mu \mathrm{m}$ ) is its modular dual column architecture, which facilitates an event-driven readout. The pixel matrix is a multiple of independent dual columns with a bi-directional shift register. Each pixel comprises a comparator and a 3bit prescaler plus overflow bit. The overflow bit starts the readout logic sequence. The pixel address is then sent off the pixel matrix and stored in a 16-bit histogram memory. This results in a practically deadtime free system, which should enable studying microsecond timescale processes. The future ASIC in $0.35 \mu \mathrm{m}$ TSMC technology will pursue the dual column architecture. The final DPAD detector is planned to cover an area of $15 \times 15 \mathrm{~cm}^{2}$ making use of a 'dual roof tile' arrangement of the detector modules.

\subsubsection{Arizona Readout}

A quite different project is described here showing a new application for quantum imaging with semiconductor detectors. This group is assessing the benefits of high-resolution semiconductor arrays for nuclear imaging and in particular for small-animal SPECT ${ }^{\text {[5 }}$ [76],[77] They use $1.5 \mathrm{~mm}$ thick CdZnTe crystals as gamma detection medium arranged in a 64 x 64 matrix of $380 \mu \mathrm{m}$ square pixels. The sensor is In bump-bonded to the Arizona Readout ASIC (Mitel $3 \mu \mathrm{m}$ technology), which consists basically of a capacitive-feedback transimpedance amplifier, a correlated double sample and hold circuit, a buffer and a shift register [78], The analogue signals are sent out to a digital signal processing board containing a 12-bit ADC and a processor. After digitisation, offset and gain correction are performed before the signal

\footnotetext{
${ }^{13}$ http://www.ideas.no/

${ }_{15}$ Digital Pixel Array Detector

${ }^{15}$ Single Photon Emission Computed Tomography
} 
gets compared to a threshold. The gamma events are then stored in the appropriate bins of a pulseheight spectrum histogram. To perform small-animal tomographic imaging a system was built consisting of a $2 \times 2 \mathrm{CdZnTe}$ detector array and a parallel-hole collimator, all mounted on a set of translation stages. The small animal, after being anaesthetised, is placed into a cylindrical holder that is attached to a rotation stage [76]. This yields a set of projection images representing the uptake distribution of the radioactive tracer. To decrease the acquisition time it is planned to construct a ring with the CdZnTe detector arrays.

\subsubsection{NexRay}

A completely new approach for X-ray diagnosis was adopted with the construction of a commercial scanning-beam digital $x$-ray (SBDX) system [79] It aims at cardiac fluoroscopy and angiography with a CdZnTe pixel detector array [80]. Instead of using a conventional point-like X-ray source and a large area detector underneath the patient, an electron beam is electro-magnetically scanned across a large $23 \times 23 \mathrm{~cm}^{2}$ transmission target in case of the SBDX system and focused through a rectangular collimator grid of $100 \times 100$ apertures onto the $5.4 \times 5.4 \mathrm{~cm}^{2} \mathrm{CdZnTe}$ detector array (see schematic drawing fig. 8 [81]. The full field of view (entire cardiac region) is scanned at up to 30 frames/s. Sixteen slices through the patient are reconstructed in real time (tomographic reconstruction based on the slightly different angle of the 10000 scan positions). This geometry naturally reduces scattering in the patient substantially; there is no need for an anti-scatter grid. Moreover, skin injury is reduced due to the larger radiation entrance area, and doctors have easier access to the patient during surgery. The CdZnTe SBDX detector is $3 \mathrm{~mm}$ thick (absorption efficiency $>90 \%$ at $120 \mathrm{kVp}$ ) and comprises $12 \times 12$ square pixels of $1.125 \mathrm{~mm}$ pitch. To simplify the photon counting readout chip, each pixel is divided into 60 binary subelements of size $225 \times 95 \mu \mathrm{m}^{2}$, which are In bump-bonded to the subchannels of the ASIC (designed by Adept IC Design, California, in Mosis $1.2 \mu \mathrm{m}$ technology). The signal gets amplified and discriminated in each subchannel; an anti-coincidence logic with the bottom and right neighbours limits double counting. Finally the outputs of the 60 subchannels are summed up to yield the total number of counts in the $1.125 \mathrm{~mm}$ pixel. $4 \times 4$ of these CdZnTe assemblies are staggered in the final detector array.

\footnotetext{
${ }^{16}$ former Cardiac Mariners
} 


\subsection{Projects based on pad detectors}

\subsubsection{DEBI}

It has been shown [82] that it is possible to characterise tissue composition with the help of a technique called energy dispersive X-ray diffraction (EDXRD), in which the scattered radiation of a poly-energetic $\mathrm{X}$-ray source is measured at a fixed angle ${ }^{18}$. For small angles $<10^{\circ} \mathrm{X}$-ray scattering is predominantly coherent, and in this case the magnitude of scatter is governed by the specific form factor. Measured scatter signatures of normal compared to cancerous breast tissue showed significant differences in shape and pulse height. The DEBI project tries to make use of this technique. In their mammography system [83] they use Si pad detectors ( $1 \mathrm{~mm}$ thick, $6 \times 21$ pads of $2 \mathrm{~mm}$ pitch) placed at small scattering angles in addition to a flat-panel detector, which records the normal transmission image. The pad modules are read out by 128-channel ASICs developed by Ideas, Norway. The chip sends out the analogue signals that pass the defined threshold to external ADCs. Transmission and diffraction images can thus be superimposed to increase the information content of the examination.

\subsection{2 $\quad$ ABIS $^{19}$}

A prototype automatic baggage inspection system is under development, for which a CdZnTe linear detector array was built [85], The detector arm is about $1 \mathrm{~m}$ long and is composed of $64 \mathrm{CdZnTe}$ detectors. The sensors are $1.75 \mathrm{~mm}$ thick and have $2 \times 16$ pads of size $1 \times 1 \mathrm{~mm}^{2}$. A 32-channel ASIC called FESA ${ }^{20}$ is used to process the signals [86] Each of the readout channels comprises a polarity input, an amplification stage with programmable and tunable gain, baseline adjustment and 5 comparators with individually selectable threshold followed by the corresponding 18-bit counters. The resulting distribution of the events within the available energy bands should enable the identification of explosive materials.

\subsubsection{Bone densitometry with CdTe arrays}

Another application where energy information is used is the development of a bone densitometer with a linear CdTe array [87]. In this system two thresholds, each connected to a 16-bit counter, are available. The bone mineral content is determined by measuring the difference of the bone absorption using two

\footnotetext{
${ }^{17}$ Diffraction Enhanced Breast Imaging

${ }^{18}$ EDXRD can be used for the identification of explosives during baggage inspection as well [84].

${ }^{19}$ Automatic Baggage Inspection System

${ }^{20}$ Front-end Electronics for Spectroscopic Applications
} 
incident radiation energies (DEXA ${ }^{21}$ method). This is obtained through offline subtraction of the upper counter value from the lower counter value. It is no longer necessary to take two separate images to perform the dual energy image subtraction, which saves time, increases the lifetime of the X-ray tube and most importantly reduces the patient dose.

\section{The Medipix Project}

The Medipix project produced the first full-scale quantum imaging ASIC for hybrid semiconductor pixel detectors [88] The readout chip called Medipix1/PCC ${ }^{22}$ that was designed within the CERN microelectronics group in the framework of the Medipix1 Collaboration [89] was based on earlier experience with the design of pixel chips for high-energy particle trackers [90][[93]. Most of the abovementioned quantum imaging projects originated in groups active in particle physics tracking. It was evident that such true 2-dimensional devices could be used to reconstruct patterns for various imaging applications as well, and for X-rays in particular [94][95].

The primary applications in view for Medipix 1 were mammography and dental radiology, but it became also a research tool to study the photon counting principle. Besides mammography [48],[96] and dental imaging [97][98] the Medipix1 chip is also being evaluated for synchrotron applications [98][99], gamma imaging [100],[101], dynamic autoradiography [47] electron microscopy [102], dynamic defectoscopy [103] and many other applications.

The Medipix1 ASIC was available in 1997 and its success encouraged the Medipix2 Collaboration [104] to initiate the design of an improved version with the name Medipix2, which came back from foundry just recently. This section will explain the project in more detail and present some unpublished measurements. The underlying principle is the same as in the projects already described, and therefore a lot of the observations are generally valid for other photon counting semiconductor detectors as well.

\subsection{The Medipix1 photon counting detector}

The architecture of the Medipix1 chip designed in $1 \mu \mathrm{m}$ SACMOS technology has been described in detail elsewhere [88] Each pixel has a preamplifier, a discriminator with 3 bits of threshold adjustment and a 15-bit counter. The threshold of each pixel cell can be determined and tuned individually with electrical pulsing, and non-functional pixels can be masked. The pixels measure $170 \times 170 \mu \mathrm{m}^{2}$ and are arranged in a $64 \times 64$ matrix. Bump-bonding to silicon as well as to GaAs sensors was carried out.

\footnotetext{
${ }^{21}$ Dual Energy X-ray Absorptometry

${ }^{22}$ Photon Counting Chip
} 
Extensive characterisation work was performed and images taken [11][12],[50],[99],[106]. An example of a Medipix1 image is shown in fig. 9 (raw data!). Moreover, an effort was made to simplify the system through hardware [107] [108] and software improvements [109].

The following subsections will explain the effect of threshold adjustment and flat field correction with Medipix1 data.

\subsubsection{The Influence of Threshold Adjustment}

The threshold settings in the individual pixels affect the quality of an image in a counting device. One of the reasons for a non-uniform threshold distribution is the area dependent mismatch between transistors. Therefore most of the above-mentioned quantum imaging chips include a threshold tuning circuit narrowing the threshold distribution of the whole chip.

Threshold adjustment is essential for applications with energies close to the minimum threshold of the chip or threshold settings close to the beam energy or when it is required to cut into the beam spectrum (e.g. with window discrimination). The first case is illustrated by a measurement done with a Medipix1 Si detector and a ${ }^{55} \mathrm{Fe}$ source producing $5.9 \mathrm{keV}$ X-rays (corresponds to $\sim 1640 \mathrm{e}^{-}$deposited charge in a silicon sensor) (fig. 10(a)). Two $\sim 100 \mu \mathrm{m}$ thick silicon objects (a triangle and one half of an octahedron) are illuminated for $5 \mathrm{~min}$ with a ${ }^{55} \mathrm{Fe}$ source. Without threshold adjustment many white pixels can be seen corresponding to non-counting pixels with thresholds above $5.9 \mathrm{keV}$. Fig. 10 (b) shows the image of the two objects taken under the same conditions, but with the 3-bit threshold tuning applied that enables an overall reduction in threshold of the pixel matrix. The advantage of this hardware facility is clearly visible. If the deposited energy of the photon beam is well above the threshold of the chip, the visible improvement is less evident. Fig. 11(a) shows the image of the same two objects without threshold adjustment, whereas threshold adjustment was applied to yield image 11(b). With the data of fig. 11(b) it is still not possible to distinguish two distributions (the shadow region below the object and the background) in a histogram of the count distribution. A ${ }^{109} \mathrm{Cd} \mathrm{X}$-ray source $e^{23}$ was used for this measurement.

It is possible to calculate the SNR of the two objects with respect to the background. The SNR is given by [111][112]

$$
S N R=\frac{N-N_{o}}{\sqrt{\sigma^{2}(N)+\sigma^{2}\left(N_{o}\right)}}
$$

\footnotetext{
${ }^{23}$ Several X-ray emission lines of ${ }^{109} \mathrm{Cd}$ are concentrated around 22 and $25 \mathrm{keV}$ [110]; the $\gamma$-ray emission line at $88 \mathrm{keV}$ can be neglected due to the low probability of conversion in the $300 \mu \mathrm{m}$ thick silicon sensor.
} 
with $N$ as the mean number of counts in the background area and $N_{o}$ as the mean number of counts in the area underneath the object. For the raw data presented in fig. 11(a), the SNR is $\sim 1$ and improves to $\sim 1.9$ when threshold adjustment is applied (fig. 11(b)).

\subsubsection{Systematic Pixel-to-Pixel Variations}

Systematic variations must be minimised in every imaging system. In the case of a semiconductor pixel detector there are various mechanisms that lead to systematic pixel-to-pixel variations. Non-uniformities in sensor material resistivity, in doping, impurity and defect level distribution, in the thickness of the sensor rear side contact or in the photolithography could result in non-uniform charge collection properties. Electronic mismatch in the readout chip does not seem to play a major role for a counting system if the threshold is set comfortably above noise and below the beam energy. Non-uniformities can evidently arise as well from non-uniform beam illumination.

The usual image correction method to reduce systematic noise is background subtraction where an image without object (a flood image) is subtracted from the image with the object present under equal imaging conditions. Fig. 12(a) shows such a background-subtracted image ${ }^{24}$ of the two silicon objects (same measurement setup as used for fig. 11). The histogram representation (fig. 12(b)) features clearly a tail at lower counts arising from the attenuation in the object. This signifies an improvement compared to the uncorrected image although there is still no confident distinction possible between two wellseparated distributions. The SNR is $\sim 2.2$ in this case.

The most efficient correction method for the suppression of systematic noise proved to be the flat field correction. The principle of this method is to produce an 'efficiency' map of the pixel detector and divide the content of each pixel counter with its corresponding flat field coefficient ${ }^{25}$. For a precise efficiency map several flood images should be added up. The flat field coefficient per pixel is then given by the ratio

$$
\frac{\text { individual pixel count }}{\text { mean number of counts }}
$$

determined from the added flood field acquisitions. Applying this correction method to the data of fig. 11(a) results in fig. 13. Two clearly separated distributions are now visible in the 1-dimensional count representation allowing a precise identification of the object area and increasing the SNR to $\sim 2.5$.

This contrast enhancement through the flat field correction is very useful to image low-contrast objects.

To illustrate this an attempt was made to image a fly (fig. 14). For a ${ }^{109} \mathrm{Cd}$ source the fly was simply

\footnotetext{
${ }^{24}$ The mean number of counts from fig. 10(a) was added to the subtracted values of each pixel.

${ }^{25}$ In this context the term 'efficiency map' should be understood as a picture of any kind of systematic variations in the entire detector system.
} 
transparent. To increase the attenuation a lower energy source $\left({ }^{55} \mathrm{Fe}\right)$ was used instead. Fig. 15(a) shows the resulting image with threshold tuning only; for image 15(b) threshold adjustment and flat field correction were combined ${ }^{26}$. This made it possible for internal structural details of the insect to emerge as well as the extremely low-contrast areas at the origin of the wings.

For counting experiments the limit of improvement for pixel-to-pixel variations of various hardware and software correction methods is given by the random quantum fluctuations of the impinging photons, which are described by Poisson statistics. Therefore the minimum standard deviation of a uniformly illuminated flood image is given by $\sqrt{N}$ (with $N$ as the mean number of impinging photons) and the highest achievable SNR by $N / \sqrt{N}$.

The SNR of a Medipix1 Si detector was extensively studied. For this purpose the detector was uniformly illuminated with X-rays from a Mo tube $(30 \mu \mathrm{m} \text { Mo filter, } 28 \mathrm{kV}, 2 \mathrm{~mA})^{27}$. With a threshold fixed at $\sim 8.5 \mathrm{keV}$ and threshold adjustment on $\left(\sigma_{\mathrm{thr}} \sim 0.5 \mathrm{keV}\right)$, a maximum SNR of $\sim 30$ could be reached (dasheddotted curve with open circles in fig. 16). The x-axis shows the normalised acquisition time and represents the full dynamic range of Medipix1 (15 bits). It is interesting to notice that the SNR quickly reaches a plateau and does not increase much anymore with increasing dose. However, by applying the flat field coefficients in the way described above, the Medipix1 SNR perfectly follows the Poisson limit (solid curve and open squares) over its full dynamic range, up to values of $\sim 160$. The dashed curves with filled squares, filled circles and filled stars correspond to different statistics used to determine the flat field coefficients (100 flood fields added, 50 and 25 , respectively) and do not differ greatly. Furthermore it was verified that the flat field for these measurements is not determined by the relative position of the detector to the source by displacing the detector for an equivalent of 2 pixels and applying the original flat field coefficients. The result remained unchanged.

The measurements were repeated increasing the threshold to about $12.4 \mathrm{keV}$, which means cutting already slightly into the Mo spectrum (compare fig. 17 113]. With threshold adjustment $\left(\sigma_{\mathrm{thr}} \sim 0.4 \mathrm{keV}\right)$, but without flat field correction, a SNR of $\sim 19$ could be reached, significantly below the one at lower threshold. Staying at about the same global threshold $(\sim 11.7 \mathrm{keV})$ without flat field correction and this time without threshold adjustment $\left(\sigma_{\mathrm{thr}} \sim 1.4 \mathrm{keV}\right)$, the SNR drops to about 7. Nevertheless, for both cases, applying the flat field correction, the SNR follows again perfectly the Poisson limit over the full dynamic range of the chip.

\footnotetext{
${ }^{26}$ For the correction map 6 flood fields were added.

${ }^{27}$ The resulting X-ray spectrum of this configuration is typical for mammographic tubes.
} 
Going back to the original configuration ( $8.5 \mathrm{keV}$ threshold, threshold adjustment mask loaded), the detector bias was increased from $35 \mathrm{~V}$ (nominal full depletion voltage for the $300 \mu \mathrm{m}$ thick sensor is $20 \mathrm{~V}$ ) to $80 \mathrm{~V}$. The resulting SNR without flat field correction stayed approximately the same $(\sim 31)$, but the mean number of counts in each acquisition decreased in average for $4.3 \%$. This indicates that working in over-depletion mode reduces charge sharing [114].[115]. Going below full depletion (17 $\mathrm{V}$ detector bias), the SNR decreased to $\sim 19$. Again a perfect agreement between the theoretical SNR limit and the measurements at different detector bias voltages was reached applying the flat field correction (even for the case of under-depletion). Analysing the $17 \mathrm{~V}$ data one point fell completely out of range (see fig. 18, second last points). These were the first measurement points taken immediately after switching on the detector. There the flat field correction did not work properly, which suggests firstly that the electric field distribution within the sensor was not yet in equilibrium (a decreasing detector leakage current seems to point to a filling of shallow traps) and secondly that the impressive improvement of the flat field correction is mainly due to inhomogeneities in the sensor and not in the readout electronics.

The $17 \mathrm{~V}$ data revealed as well an interesting feature of a silicon sensor (observed with several detectors), bands of higher and lower charge collection efficiency (see top fig. 19(a)). This fact is due to radial doping non-uniformities during crystal growth and is well known in literature [116][117]. Applying the flat field correction this pattern disappears and the image becomes perfectly uniform (bottom fig. 19(a)). The bands disappear as well after full depletion of the sensor. Fig. 19(b) (top) shows a flood image at $80 \mathrm{~V}$ detector bias. Applying the wrong flat field map (the one from the $17 \mathrm{~V}$ data) to this image, it is evident that the result is the inverse band structure (bottom fig. 19(b)) and that the SNR degrades strongly. Nevertheless the very interesting observation could be made that the local pixel-to-pixel variations improved considerably. This indicates a fixed pattern noise, which is independent of the applied detector bias. One can also state that this fixed pattern noise is stable as the flat field correction works perfectly well. The origin of this fixed pattern noise is still the subject of investigation.

A further experiment to determine the dependence of the flat field correction on the incoming spectrum was set up again with a Mo tube (30 $\mu \mathrm{m}$ Mo filter, $28 \mathrm{kV})$ and a Medipix1 Si detector. Flood field images were taken, adding one or more layers of $1 \mathrm{~cm}$ thick Perspex (up to four layers). This results in a hardening of the spectrum and is similar to the situation given in mammography with different breast thickness. In fig. 20 the standard deviation of the flood images normalised to the Poisson limit is plotted for different thicknesses of Perspex. The solid curve corresponds to the standard deviation obtained without flat field correction. The dashed curves relate measurement points, which were corrected with the flat field coefficients obtained from flood fields after a certain Perspex layer thickness (e.g. squares 
signify a correction with the flat field map determined without Perspex layer). Fig. 20 therefore shows that the improvement with the flat field correction degrades with the difference in incident spectrum, and in the end the non-corrected image is better than the corrected one (flood fields after 3 and $4 \mathrm{~cm}$ Perspex corrected with the 0-layer flat field map). To deal with the energy-dependence of the flat field correction, it has been shown [118] that interpolated flat field maps can be used, which correct almost down to the Poisson limit. Measurements and simulations are being prepared to investigate more in detail the origin of this energy dependence.

All these measurements show that a quantum imaging detector of small pixel size like the Medipix1 detector can be used as a tool to investigate sensor properties and that the flat field correction is a quite simple, but extremely powerful correction method for stable pixel-to-pixel non-uniformities.

\subsection{The Medipix2 chip}

The main purpose of the Medipix2 chip was to decrease the pixel size in order to become competitive in terms of spatial resolution with other imaging detectors and at the same time to increase functionality. This goal could be achieved using a $0.25 \mu \mathrm{m}$ CMOS technology. Medipix 2 is for the moment the quantum imaging hybrid pixel detector with the smallest pixel size, which should help to answer many open questions concerning charge sharing or minimum pixel sizes needed for certain applications. For example for mammography it could contribute data to help understand whether a pixel size of $100 \mu \mathrm{m}$ is sufficient or whether a smaller pixel size could detect earlier phases in the formation of microcalcifications and therefore increase the chance of earlier breast cancer detection.

A detailed description of the Medipix2 chip can be found in [119] It is arranged in a $256 \times 256$ pixel matrix (65536 pixels/chip!) and measures $\sim 1.4 \times 1.6 \mathrm{~cm}^{2}$. Each pixel is square with $55 \mu \mathrm{m}$ on the side and accepts positive as well as negative charge input signals. The block diagram of the pixel cell is shown in fig. 21. Each cell contains a charge preamplifier with individual leakage current compensation (implemented after the Medipix1 experience with GaAs sensors) and a window discriminator. The double discriminator logic identifies charge signals, which lie between the lower and upper threshold, and increments the 13-bit counter. Each of the two discriminators can be fine-tuned with 3 bits, and each pixel can be masked and tested. The pixels are designed to work up to a count rate of $1 \mathrm{MHz}$, which provides the highest counting rate per unit area available at present. Special care has been taken to enable covering of larger detection areas without dead space. Therefore dead areas at 3 sides of the chip were minimised. A $2 \times 4$ chipboard was designed allowing a sensitive area coverage of $\sim 2.8 \mathrm{x} \sim 5.6 \mathrm{~cm}^{2}$. This module will have more than $500 \mathrm{k}$ pixels. 
First tests show that both the analog and the digital part of the ASIC work as expected. The window logic is functional as well as the threshold adjustments. A first image generated by loading a mask and pulsing the chip with 1000 pulses is presented in fig. 22. The matrix was unadjusted and the threshold set to about $4000 \mathrm{e}^{-}$; the test pulse height corresponded to $\sim 8000 \mathrm{e}^{-}$.

Bump-bonding to thin and thick silicon as well as to CdTe sensors is planned for the near future. The benefit of window discrimination for various applications will be a major field of activity. Moreover, it should be possible with these detectors to investigate the limits of quantum imaging with regard to charge sharing. Depending on the aspect ratio of pixel area to sensor thickness as well as on the field strength, charge sharing effects will certainly become an issue. Some new ideas for a novel pixel cell architecture have already been presented in [119] to tackle this problem, but the effect should be extensively studied beforehand. A $3 \times 3$ pixel array located in the Medipix2 periphery with analog output is foreseen for this purpose.

\section{Conclusions and Trends}

In this paper the state of the art of quantum imaging with segmented semiconductor detectors has been reviewed. A selection of quantum imaging projects was presented with the Medipix project in more detail. The consequences of basic image correction methods and in particular of the flat field correction have been illustrated with new images. It has been shown that such a hybrid photon counting pixel detector can be a powerful tool to study the connected sensor material itself, especially with the steadily decreasing pixel size possible thanks to the fast progress in microelectronics.

From the overview of the projects it can be observed that a large fraction of the projects have their origins in high-energy physics. Most of the projects focus on a specific imaging task except perhaps the Medipix project, which investigates the detectors for various applications. Over the last years groups from completely new application fields started to show interest in this development, and it is evident that there is still an enormous, undiscovered potential for new areas of application. The full potential of quantum imaging with semiconductor detectors is only partly explored as yet.

Although there has been some major progress in large area coverage, there is still no cost-effective solution available at present for standard radiological equipment.

There is a clear trend away from simple photon counting towards a more sophisticated characterisation of the $\mathrm{x}$-rayed object. For this purpose energy information is required. Therefore most of the future quantum imaging chips will at least possess an energy windowing facility or even a multi-bit ADC per readout channel. Based on these developments, 'colour' X-ray imaging will become a reality. 


\section{Acknowledgements}

The author would like to specially thank the various mentioned projects for providing her with information.

The steady support of the Medipix Collaboration and the CERN microelectronics group is warmly acknowledged. Particular thanks for their contribution to this paper to Michael Campbell, Erik Heijne, Lukas Tlustos, Xavier Llopart, Birgit Schatz and Mokhtar Chmeissani. 


\section{Bibliography}

[1] J. Kemmer, P. Burger, R. Henck and E. Heijne, Performance and applications of passivated ionimplanted silicon detectors, IEEE Trans. Nucl. Sci. NS-29 (1982) 733-737

[2] J. Kemmer, Improvement of Detector Fabrication by the Planar Process, NIM 226 (1984) 89-93

[3] http://physics.nist.gov/PhysRefData/XrayMassCoef/cover.htm

[4] C.M. Buttar, GaAs detectors - A review, NIM A 395 (1997) 1-8

[5] T.E. Schlesinger and R.B. James, editors, Semiconductors for Room Temperature Nuclear Detector Applications, vol. 43, Academic Press 1995

[6] D.S. McGregor and H. Hermon, Room-temperature compound semiconductor radiation detectors, NIM A 395 (1997) 101-124

[7] F. Nava, G. Bertuccio, P. Vanni, C. Canali, A. Cavallini, A. Castaldini and L. Polenta, Improved Performance of GaAs Radiation Detectors with Low Temperature Ohmic Contacts, IEEE Trans. Nucl. Sci., vol. 44, no. 3, June 1997, 943-949

[8] R.L. Bates, M. Campbell, S. D’Auria, C. Da Vià, V. O'Shea, C. Raine and K.M. Smith, Recent results on GaAs detectors, NIM A 392 (1997) 269-273

[9] E. Bertolucci, M. Conti, G. Mettivier, P. Russo, A. Cola, F. Quaranta, L. Vasanelli, M.G. Bisogni, U. Bottigli, M.E. Fantacci and A. Stefanini, Spectroscopic performance of semi-insulating GaAs detectors for digital radiography, NIM A 422 (1999) 247-251

[10] J. Darmo, F. Dubecký, B. Zat'ko, P. Boháček, M. Sekáčová, J. Kvitkovič, V. Nečas and P.G. Pelfer, The semi-insulating GaAs-based particle detector at IEE SAS: first imaging results, NIM A 458 (2001) 418-421

[11] C. Schwarz, M. Campbell, R. Göppert, J. Ludwig, B. Mikulec, K. Runge, K.M. Smith and W. Snoeys, Measurements with Si and GaAs pixel detectors bonded to photon counting readout chips, NIM A $466(2001) 87-94$

[12] B. Mikulec, Single Photon Detection with Semiconductor Pixel Arrays for Medical Imaging Applications, PhD thesis, University of Vienna, Austria, June 2000, CERN-THESIS-2000-021, 2000

[13] J.E. Eberhardt, R.D. Ryan and A.J. Tavendale, Evaluation of Epitaxial n-GaAs for Nuclear Radiation Detection, NIM 94 (1971) 463-476

[14] T. Kobayashi, T. Sugita, M. Koyama and S. Takayanagi, Performance of GaAs Surface-Barrier Detectors Made from High-Purity Gallium Arsenide, IEEE Trans. Nucl. Sci., vol. 19, no. 3, 1972, 324333 
[15] G. Bertuccio, A. Pullia, J. Lauter, A. Förster and H. Luth, Pixel X-ray Detectors in Epitaxial Gallium Arsenide with High-Energy Resolution Capabilities (Fano Factor Experimental Determination), IEEE Trans. Nucl. Sci., vol. 44, no. 1, February 1997, 1-5

[16] R.L. Bates, S. Manolopoulos, K. Mathieson, A. Meikle, V. O'Shea, C. Raine, K.M. Smith, J. Watt, C. Whitehill, S. Pospíšil, I. Wilhelm, Z. Doležal, H. Juergensen and M. Heuken, Development of lowpressure vapour-phase epitaxial GaAs for medical imaging, NIM A 434 (1999) 1-13

[17] A. Owens, M. Bavdaz, S. Kraft, A. Peacock, R. Strade, S. Nenonen, H. Andersson, M.A. Gagliardi, T. Gagliardi and H. Graafsma, Synchrotron characterization of deep depletion epitaxial GaAs detectors, J. Appl. Phys., vol. 86, no. 8, 15 October 1999, 4341-4347

[18] A. Owens, M. Bavdaz, A. Peacock, A. Poelaert, H. Andersson, S. Nenonen, H. Sipila, L. Tröger and G. Bertuccio, High resolution x-ray spectroscopy using GaAs arrays, J. Appl. Phys., vol. 90, no. 10, 15 November 2001, 5376-5381

[19] J.C. Bourgoin, N. de Angelis, K. Smith, R. Bates, C. Whitehill and A. Meikle, Potential of thick GaAs epitaxial layers for pixel detectors, NIM A 458 (2001) 344-347

[20] H. Samic, G.C. Sun, V. Donchev, N.X. Nghia, M. Gandouzi, M. Zazoui, J.C. Bourgoin, H. El-Abbassi, S. Rath and P.J. Sellin, Characterization of thick epitaxial GaAs layers for X-ray detection, NIM A 487 (2002) 107-112

[21] J.E. Toney, T.E. Schlesinger and R.B. James, Optimal bandgap variants of $\mathrm{Cd}_{1-x} \mathrm{Zn}_{\mathrm{x}} \mathrm{Te}_{\mathrm{e}}$ for highresolution X-ray and gamma-ray spectroscopy, NIM A 428 (1999) 14-24

[22] Y. Eisen, A. Shor and I. Mardor, CdTe and CdZnTe gamma ray detectors for medical and industrial imaging systems, NIM A 428 (1999) 158-170

[23] C. Scheiber and G.C. Giakos, Medical applications of CdTe and CdZnTe detectors, NIM A 458 (2001) 12-25

[24] B.A. Brunett, J.M. Van Scyoc, T.E. Schlesinger and R.B. James, The spatial response of CdZnTe gamma-ray detectors as measured by gamma-ray mapping, NIM A 458 (2001) 76-84

[25] M.R. Squillante, L. Cirignano, R. Grazioso, Room-temperature semiconductor device and array configurations, NIM A 458 (2001) 288-296

[26] J.M. Van Scyoc, B.A. Brunett, T.E. Schlesinger and R.B. James, Further investigation of the operation of $\mathrm{Cd}_{1-\mathrm{x}} \mathrm{Zn}_{\mathrm{x}}$ Te pixel array detectors, NIM A 458 (2001) 310-318

[27] T. Takahashi, S. Watanabe, M. Kouda, G. Sato, Y. Okada, S. Kubo, Y. Kuroda, M. Onishi and R. Ohno, High-Resolution CdTe Detector and Applications to Imaging Devices, IEEE Trans. Nucl. Sci., vol. 48, no. 3, June $2001,287-291$ 
[28] A. Owens, M. Bavdaz, H. Andersson, T. Gagliardi, M. Krumrey, S. Nenonen, A. Peacock, I. Taylor and L. Tröger, The X-ray response of CdZnTe, NIM A 484 (2002) 242-250

[29] C.M.H. Chen, S.E. Boggs, A.E. Bolotnikov, W.R. Cook, F.A. Harrison and S.M. Schindler, Numerical Modeling of Charge Sharing in CdZnTe Pixel Detectors, IEEE Trans. Nucl. Sci., vol. 49, no. 1, February 2002, 270-276

[30] S. Yin, T.O. Tümer, D. Maeding, J. Mainprize, G. Mawdsley, M.J. Yaffe, E.E. Gordon and W.J. Hamilton, Direct Conversion CdZnTe and CdTe Detectors for Digital Mammography, IEEE Trans. Nucl. Sci., vol. 49, no. 1, February 2002, 176-181

[31] K. Hitomi, O. Muroi, M. Matsumoto, R. Hirabuki, T. Shoji, T. Suehiro and Y. Hiratate, Recent progress in thallium bromide detectors for X- and $\gamma$-ray spectroscopy, NIM A 458 (2001) 365-369

[32] A. Owens, M. Bavdaz, I. Lisjutin, A. Peacock, H. Sipila and S. Zatoloka, On the development of compound semiconductor thallium bromide detectors for astrophysics, NIM A 458 (2001) 413-417

[33] P.G. Pelfer, F. Dubecký, R. Fornari, M. Pikna, E. Gombia, J. Darmo, M. Krempaský and M. Sekáčová, Present status and perspectives of the radiation detectors based on InP materials, NIM A 458 (2001) 400-405

[34] A. Owens, M. Bavdaz, V. Gostilo, D. Gryaznov, A. Loupilov, A. Peacock and H. Sipila, The X-ray response of InP, NIM A 487 (2002) 435-440

[35] M. Schieber, H. Hermon, A. Zuck, A. Vilensky, L. Melekhov, R. Shatunovsky, E. Meerson and H. Saado, Theoretical and experimental sensitivity to X-rays of single and polycrystalline $\mathrm{Hgl}_{2}$ compared with different single-crystal detectors, NIM A 458 (2001) 41-46

[36] L. van den Berg and R.D. Vigil, Fabrication of mercuric iodide radiation detectors, NIM A 458 (2001) $148-151$

[37] J.S. Iwanczyk, B.E. Patt, C.R. Tull, L.R. MacDonald, N. Skinner, E.J. Hoffman and L. Fornaro, Hgl 2 Polycrystalline Films for Digital X-Ray Imagers, IEEE Trans. Nucl. Sci., vol. 49, no. 1, February $2002,160-164$

[38] T. Shoji, K. Ohba, T. Suehiro and Y. Hiratate, Fabrication of Radiation Detector Using $\mathrm{Pbl}_{2} \mathrm{Crystal}_{\text {, }}$ IEEE Trans. Nucl. Sci., vol. 42, no. 4, August 1995, 659-662

[39] K.S. Shah, R.A. Street, Y. Dmitriyev, P. Bennett, L. Cirignano, M. Klugerman, M.R. Squillante and G. Entine, X-ray imaging with $\mathrm{Pbl}_{2}$-based a-Si:H flat panel detectors, NIM A 458 (2001) 140-147

[40] J.V. Vaitkus, R. Irsigler, J. Andersen and K.M. Smith, Instabilities in LEC GaAs Schottky barrier pixel detector imaging arrays, NIM A 460 (2001) 204-206 
[41] G.E. Moore, Cramming More Components onto Integrated Circuits, Electronics, vol. 38, no. 8, 19 April 1965, 114-117

[42] G.E. Moore, Progress in Digital Integrated Electronics, Technical Digest of the IEEE International Electron Device Meeting 1975, 11-13

[43] http://public.itrs.net/Files/2001/TRS/Home.htm, International Technology Roadmap for Semiconductors 2001

[44] M. Campbell, G. Anelli, E. Cantatore, F. Faccio, E.H.M. Heijne, P. Jarron, J.-C. Santiard, W. Snoeys and K. Wyllie, An introduction to deep submicron CMOS for vertex applications, NIM A 473 (2001) $140-145$

[45] R.N. Cahn, B. Cederström, M. Danielsson, A. Hall, M. Lundqvist and D. Nygren, Detective quantum efficiency dependence on x-ray energy weighting in mammography, Med. Phys. 26 (12), December $1999,2680-2683$

[46] M. Lundqvist, B. Cederström, V. Chmill, M. Danielsson and B. Hasegawa, Evaluation of a PhotonCounting X-Ray Imaging System, IEEE Tans. Nucl. Sci., vol. 48, no. 4, August 2001, 1530-1536

[47] E. Bertolucci, M. Conti, A. Di Cosmo, M. Maiorino, G. Mettivier, M.C. Montesi, G. Paolella, T. Pecorella, P. Russo and R. Scognamiglio, Real time $\beta$-imaging with silicon hybrid pixel detectors: investigations into amino acids uptake and genetics, IEEE NSS/MIC Conference, San Diego, California, 4-10 November 2001, Conf. Records M13A-24

[48] S.R. Amendolia, M.G. Bisogni, U. Bottigli, M.A. Ciocci, P. Delogu, G. Dipasquale, M.E. Fantacci, P. Maestro, V. Marzulli, B. Mikulec, E. Pernigotti, V. Rosso, A. Stefanini and S. Stumbo, Test of a GaAs-based pixel device for digital mammography, NIM A 460 (2001) 50-54

[49] C. Schwarz, M. Campbell, R. Göppert, J. Ludwig, B. Mikulec, M. Rogalla, K. Runge, A. SöldnerRembold, K.M. Smith, W. Snoeys and J. Watt, Dose-dependent X-ray measurements using a 64x64 hybrid GaAs pixel detector with photon counting, NIM A 460 (2001) 91-96

[50] C Ponchut and F. Zontone, Evaluation of Medipix-1 in X-ray scattering and X-ray diffraction applications, see these proceedings

[51] F. Arfelli, V. Bonvicini, A. Bravin, G. Cantatore, E. Castelli, M. Fabrizioli, R. Longo, A. Olivo, S. Pani, D. Pontoni, P. Poropat, M. Prest, A. Rashevsky, L. Rigon, G. Tromba, A. Vacchi and E. Vallazza, A multiplayer edge-on silicon microstrip single photon counting detector for digital mammography, Nuclear Physics B (Proc. Suppl.) 78 (1999) 592-597

[52] F. Arfelli, G. Barbiellini, V. Bonvicini, A. Bravin, G. Cantatore, E. Castelli, L. Dalla Palma, M. Di Michiel, R. Longo, A. Olivo, S. Pani, D. Pontoni, P. Poropat, M. Prest, R. Rosei, M. Sessa, G. 
Tromba and A. Vacchi, Digital Mammography at the Trieste Synchrotron Light Source, IEEE Trans. Nucl. Sci., vol. 43, no. 3, June 1996, 2061-2067

[53] F. Arfelli, V. Bonvicini, A. Bravin, P. Burger, G. Cantatore, E. Castelli, M. Di Michiel, R. Longo, A. Olivo, S. Pani, D. Pontoni, P. Poropat, M. Prest, A. Rashevsky, G. Tromba, A. Vacchi and N. Zampa, Design and evaluation of AC-coupled, FOXFET-biased, 'edge-on' silicon strip detectors for X-ray imaging, NIM A 385 (1997) 311-320

[54] G. Comes, F. Loddo, Y. Hu, J. Kaplon, F. Ly, R. Turchetta, V. Bonvicini and A. Vacchi, CASTOR a VLSI CMOS mixed analog-digital circuit for low noise multichannel counting applications, NIM A 377 (1996) 440-445

[55] C. Colledani, G. Comes, W. Dulinski, Y. Hu, F. Loddo, R. Turchetta, V. Bonvicini, E. Castelli, D. Pontoni, M. Prest, A. Rashevsky and A. Vacchi, Castor 1.0, a VLSI analog-digital circuit for pixel imaging applications, NIM A 395 (1997) 435-442

[56] A. Bergamaschi et al., FROST : An ASIC for digital mammography with Synchrotron Radiation, see these proceedings

[57] B. Hilt, P. Fessler and G. Prévot, New quantum detection system for very low dose X-ray radiology, NIM A $442(2000) 38-44$

[58] B. Hilt, P. Fessler and G. Prévot, The quantum X-ray radiology apparatus, NIM A 442 (2000) 355359

[59] P. Fessler, J. Coffin, H. Eberlé, C. de Raad Iseli, B. Hilt, D. Huss, F. Krummenacher, J.R. Lutz, G. Prévot, A. Renouprez, M.H. Sigward, B. Schwaller and C. Voltolini, An important step forward in continuous spectroscopic imaging of ionising radiations using ASICs, NIM A 421 (1999) 130-141

[60] M. Lundqvist, private communication

[61] http://www.te.rl.ac.uk/med/projects/main.htm (X-ray_detection; Diffex)

[62] Courtesy of R. Turchetta, Rutherford lab, UK

[63] P. Delpierre, J.F. Berar, L. Blanquart, B. Caillot, J.C. Clemens and C. Mouget, X-Ray Pixel Detector for Crystallography, IEEE Trans. Nucl. Sci., vol. 48, no. 4, August 2001, 987-991

[64] P. Delpierre, J.F. Berar, L. Blanquart, N. Boudet, P. Breugnon, B. Caillot, J.C. Clemens, C. Mouget, R. Potheau and I. Valin, Large surface X-ray pixel detector, IEEE NSS/MIC Conference, San Diego, California, 4-10 November 2001, Conf. Records N23-2

[65] P. Delpierre, private communication

[66] Ch. Brönnimann et al., see these proceedings 
[67] Ch. Brönnimann, R. Baur, E.F. Eikenberry, S. Kohout, M. Lindner, B. Schmitt and R. Horisberger, A pixel read-out chip for the PILATUS project, NIM A 465 (2001) 235-239

[68] P. Seller, G. Derbyshire, G. Hall, G.M. Iles, A. Jordan, P. Murray, M.L. Prydderch, S.M. Passmore, K. Smith and S.L. Thomas, Photon counting hybrid pixel detector for X-ray imaging, NIM A 455 (2000) 715-720

[69] G. Iles, K. Mathieson, P. Murray, S. Passmore, M. Prydderch, P. Seller and S. Thomas, Large-area pixellated photon counting X-ray imaging system, NIM A 458 (2001) 427-430

[70] H. Krüger, P. Fischer, S. Krimmel, M. Lindner, M. Löcker, N. Wermes, M. Koudag, G. Sato, T. Takahashi and S. Watanabe, A X-ray Imaging System using a Single Photon Counting CdTe Pixel Detector, presentation at the IEEE NSS/MIC Conference, San Diego, California, 4-10 November 2001

[71] P. Fischer, M. Kouda, H. Krüger, M. Lindner, G. Sato, T. Takahashi, S. Watanabe and N. Wermes, A Counting CdTe Pixel Detector for Hard X-Ray and $\gamma$-Ray Imaging, IEEE Trans. Nucl. Sci., vol. 48, no. 6 , December 2001, 2401-2404

[72] M. Lindner, L. Blanquart, P. Fischer, H. Krüger and N. Wermes, Medical X-ray imaging with energy windowing, NIM A 465 (2001) 229-234

[73] http://www4.tsl.uu.se/ dixi//

[74] L. del Risco Norrlid, C. Rönnqvist, K. Fransson, R. Brenner, L. Gustafsson, F. Edling and S. Kullander, Calculation of the modulation transfer function for the X-ray imaging detector DIXI using Monte Carlo simulation data, NIM A 466 (2001) 209-217

[75] P. Datte, J.-F. Beche, F. Druillole, P.F. Manfredi, J. Millaud and N.-H. Xuong, An Overview of the DPAD Detector for Protein Crystallography, Proc. of SPIE, Detectors for Crystallography and Diffraction Studies at Synchrotron Sources, 19 July 1999, Denver, Colorado, vol. 3774, 17-29

[76] G.A. Kastis, M.C. Wu, S.J. Balzer, D.W. Wilson, L.R. Furenlid, G. Stevenson, H.B. Barber, H.H. Barrett, J.M. Woolfenden, P. Kelly and M. Appleby, Tomographic Small-Animal Imaging Using a High-Resolution Semiconductor Camera, IEEE Trans. Nucl. Sci., vol. 49, no. 1, February 2002, 172175

[77] G.A. Kastis, H.B. Barber, H.H. Barrett, S.J. Balzer, D. Lu, D.G. Marks, G. Stevenson, J.M. Woolfenden, M. Appleby and J. Tueller, Gamma-Ray Imaging Using a CdZnTe Pixel Array and a High-Resolution, Parallel-Hole Collimator, IEEE Trans. Nucl. Sci., vol. 47, no. 6, December 2000, 1923-1927 
[78] H.B. Barber, H.H. Barrett, F.L. Augustine, W.J. Hamilton, B.A. Apotovsky, E.L. Dereniak, F.P. Doty, J.D. Eskin, J.P. Garcia, D.G. Marks, K.J. Matherson, J.M. Woolfenden and E.T. Young, Development of a 64 × $64 \mathrm{CdZnTe}$ Array and Associated Readout Integrated Circuit for Use in Nuclear Medecine, Journal of Electronic Materials, vol. 26, no. 6, 1997, 765-772

[79] E.G. Solomon, B.P. Wilfley, M.S. Van Lysel, A.W. Joseph and J.A. Heanue, Scanning-beam digital x-ray (SBDX) system for cardiac angiography, SPIE Conference on Physics of Medical Imaging, San Diego, California, February 1999, SPIE vol. 3659, 246-257

[80] J.A. Heanue, D.A. Pearson and R.E. Melen, CdZnTe detector array for a scanning-beam digital xray (SBDX) system, SPIE Conference on Physics of Medical Imaging, San Diego, California, February 1999, SPIE vol. 3659, 718-725

[81] Courtesy of E. Solomon, Los Gatos, California

[82] G. Kidane, R.D. Speller, G.J. Royle and A.M. Hanby, X-ray scatter signatures for normal and neoplastic breast tissues, Phys. Med. Biol. 44 (1999) 1791-1802

[83] D.G. Darambara, R.D. Speller and P. Sellin, Development of a Dual Detector System Based on aSi:H Arrays and Multi-element Silicon Detectors for Diffraction Enhanced Breast Imaging, IEEE NSS/MIC Conference, San Diego, California, 4-10 November 2001, Conf. Records N12-3

[84] C.H. Malden and R.D. Speller, A CdZnTe array for the detection of explosives in baggage by energy-dispersive X-ray diffraction signatures at multiple scatter angles, NIM A 449 (2000) 408-415

[85] T.O. Tümer, M. Clajus, G.I. Visser, S. Yin, P.D. Willson, L. D’Aries, K.B. Parnham, B. Glick, J.L. Perry, T. Gamble, G. Creede, E. Worthington, J. Sparling, D. Maeding and D. Gorzen, Preliminary Results Obtained from a Novel CdZnTe Pad Detector and Readout ASIC Developed for an Automatic Baggage Inspection System, IEEE NSS/MIC Conference, Lyon, France, 15-20 October 2000, Conf. Records 4-36

[86] M. Clajus, T.O. Tümer, G.J. Visser, S. Yin, P.D. Willson and D.G. Maeding, Front-End Electronics for Spectroscopy Applications (FESA) IC, IEEE NSS/MIC Conference, Lyon, France, 15-20 October 2000, Conf. Records 9-227

[87] S. Baba, K. Ohmori, Y. Mito, T. Tanoue, S. Yano, K. Tokumori, F. Toyofuku and S. Kanda, Recent development of radiation measurement instrument for industrial and medical applications, NIM A 458 (2001) 262-268

[88] M. Campbell, E.H.M. Heijne, G. Meddeler, E. Pernigotti and W. Snoeys, Readout for a 64 x 64 Pixel Matrix with 15-bit Single Photon Counting, IEEE Trans. Nucl. Sci. 45 (3), June 1998, 751-753 
[89] CERN, the University of Freiburg, University of Glasgow and the University and INFN of Pisa were the founding members of the Medipix1 Collaboration.

[90] F. Anghinolfi et al., A 1006 Element Hybrid Silicon Pixel Detector with Strobed Binary Output, CERN/ECP 91-26 (1991)

[91] H. Beker, W. Beusch, M. Campbell, M.G. Catanesi, E. Chesi, J.C. Clemens, P. Delpierre, D. Di Bari, E.H.M. Heijne, P. Jarron, V. Lenti, V. Manzari, M. Morando, F. Navach, C. Neyer, F. Pengg, R. Perego, M. Pindo, E. Quercigh, N. Redaelli, D. Sauvage, G. Segato and S. Simone, A hybrid silicon pixel telescope tested in a heavy-ion experiment, NIM A 332 (1993) 188-201

[92] M. Campbell, F. Antinori, H. Beker, W. Beusch, E. Chesi, E.H.M. Heijne, J. Heuser, P. Jarron, T. Karttaavi, F. Krummenacher, L. Lopez, G. Meddeler, A. Menetrey, P. Middelkamp, C. Neyer, F. Pengg, M. Pindo, E. Quercigh, S. Simone and H. Verweij, Development of a pixel readout chip compatible with large area coverage, NIM A 342 (1994) 52-58

[93] E.H.M. Heijne et al., LHC1: A Semiconductor Pixel Detector Readout Chip with Internal, Tunable Delay Providing a Binary Pattern of Selected Events, NIM A 383 (1996) 55-63

[94] E.H.M. Heijne and P. Jarron, Development of Silicon Pixel Detectors: an Introduction , NIM A 275 (1989) 467-471

[95] E.H.M. Heijne, Imaging with 2D and 3D integrated semiconductor detectors using VLSI technology, Physica Medica Vol. IX, N. 2-3, April-September 1993, 109-116

[96] S.R. Amendolia, M.G. Bisogni, U. Bottigli, M.A. Ciocci, P. Delogu, G. Dipasquale, M.E. Fantacci, M. Giannelli, P. Maestro, V.M. Marzulli, E. Pernigotti, V. Rosso, A. Stefanini and S. Stumbo, Low Contrast Imaging With A GaAs Pixel Digital Detector, IEEE Trans. Nucl. Sci., vol. 47, no. 4, August $2000,1478-1482$

[97] C. Schwarz, M. Campbell, R. Göppert, E.H.M. Heijne, J. Ludwig, G. Meddeler, B. Mikulec, E. Pernigotti, M. Rogalla, K. Runge, A. Söldner-Rembold, K.M. Smith, W. Snoeys and J. Watt, X-ray Imaging Using a Hybrid Photon Counting GaAs Pixel Detector, Nucl. Phys. B (Proc. Suppl.) 78 (1999) 491-496

[98] J. Watt, R. Bates, M. Campbell, K. Mathieson, B. Mikulec, V. O'Shea, M.-S. Passmore, C. Schwarz, K.M. Smith and C. Whitehill, Applications of pixellated GaAs X-ray detectors in a synchrotron radiation beam, NIM A 460 (2001) 185-190

[99] C. Ponchut, J.L. Visschers, A. Fornaini, H. Graafsma, M. Maiorino, G. Mettivier and D. Calvet, Evaluation of a photon-counting hybrid pixel detector array with a synchrotron X-ray source, NIM A 484 (2002) 396-406 
[100] O. Gal, B. Mikulec, M. Million, Experimental tests of a hybrid pixellated detector for gamma imaging, NIM A 460 (2001) 113-118

[101] E. Manach, O. Gal, Simulation of single-event energy-deposition spreading in a hybrid pixellated detector for $\gamma$ imaging, NIM A 487 (2002) 142-150

[102] A.R. Faruqi and D.M. Cattermole, Digital detectors for electron microscopy, NIM A 478 (2002) 8894

[103] D. Vavrik, J. Jakubek, J. Visschers, S. Pospisil, C. Ponchut and J. Zemankova, First tests of a Medipix-1 pixel detector for X-ray dynamic defectoscopy, NIM A 487 (2002) 216-223

[104] The Medipix2 Collaboration consists of 15 European institutes; see http://medipix.web.cern.ch/MEDIPIX/

[105] B. Mikulec, M. Campbell, G. Dipasquale, Ch. Schwarz and J. Watt, Characterisation of a single photon counting pixel system for imaging of low-contrast objects, NIM A 458 (2001) 352-359

[106] S.R. Amendolia, M.G. Bisogni, U. Bottigli, P. Delogu, G. Dipasquale, M.E. Fantacci, A. Marchi, V.M. Marzulli, M. Novelli, P. Oliva, R. Palmiero, V. Rosso, A. Stefanini, S. Stumbo and S. Zucca, Imaging and Spectroscopic Performances for a Si Based Detection System, IEEE NSS/MIC Conference, Lyon, France, 15-20 October 2000, Conf. Records 4-123

[107] G. Bardelloni, E. Bertolucci, A.L.J. Boerkamp, D. Calvet, M. Conti, M. Maiorino, P. Russo and J.L. Visschers, A New Read-out System for an Imaging Pixel Detector, IEEE NSS/MIC Conference, Lyon, France, 15-20 October 2000, Conf. Records 12-57

[108] E. Bertolucci, T. Boerkamp, M. Maiorino, G. Mettivier, M.C. Montesi and P. Russo, Portable system for imaging of $\alpha, \beta$, and X-ray sources with silicon pixel detectors and Medipix1 read out, IEEE NSS/MIC Conference, San Diego, California, 4-10 November 2001, Conf. Records N22-36

[109] E. Bertolucci, M. Maiorino, G. Mettivier, M.C. Montesi and P. Russo, Medisoft4: A Software Procedure for the Control of the Medipix2 Readout Chip, IEEE NSS/MIC Conference, San Diego, California, 4-10 November 2001, Conf. Records N22-37

[110] http://nucleardata.nuclear.lund.se/database/nudat/

[111] F. Arfelli, G. Barbiellini, G. Cantatore, E. Castelli, L. Dalla Palma, M. Di Michiel, R. Longo, P. Poropat, R. Rosei, M. Sessa, G. Tromba and A. Vacchi, Silicon Detectors for Synchrotron Radiation Digital Mammography, NIM A 360 (1995) 283-286

[112] M.J. Tapiovaara and R.F. Wagner, SNR and DQE Analysis of Broad Spectrum X-Ray Imaging, Phys. Med. Biol. 30(6) (1985) 519-529 
[113] J.M. Bone, T.R. Fewell and R.J. Jennings, Molybdenum, rhodium and tungsten anode spectral models using interpolating polynomials with application to mammography, Med. Phys. 24 (12), December 1997, 1863-1873

[114] H.G. Spieler and E.E. Haller, Assessment of Present and Future Large-Scale Semiconductor Detector Systems, IEEE Trans. Nucl. Sci., vol. NS-32, no. 1, February 1985, 419-426

[115] M. Chmeissani and B. Mikulec, Performance limits of a single photon counting pixel system, NIM A $460(2001) 81-90$

[116] W. von Ammon and H. Herzer, The Production and Availability of High Resistivity Silicon for Detector Application, NIM 226 (1984) 94-102

[117] D. Nouais, M. Bondila, V. Bonvicini, P. Cerello, E. Crescio, P. Giubellino, R. Hernández-Montoya, A. Kolojvari, L.M. Montaño, B.S. Nilsen, C. Piemonte, A. Rashevsky, F. Tosello, A. Vacchi and R. Wheadon, Correction of dopant concentration fluctuation effects in silicon drift detectors, NIM A 461 (2001) 222-225

[118] David W. Davidson, University of Glasgow, to be published

[119] X. Llopart, M. Campbell, R. Dinapoli, D. San Segundo and E. Pernigotti, Medipix2, a 64k pixel readout chip with $55 \mu \mathrm{m}$ square elements working in single photon counting mode, IEEE NSS/MIC Conference, San Diego, California, 4-10 November 2001, Conf. Records M7-3, accepted for publication in IEEE Trans. Nucl. Sci. 


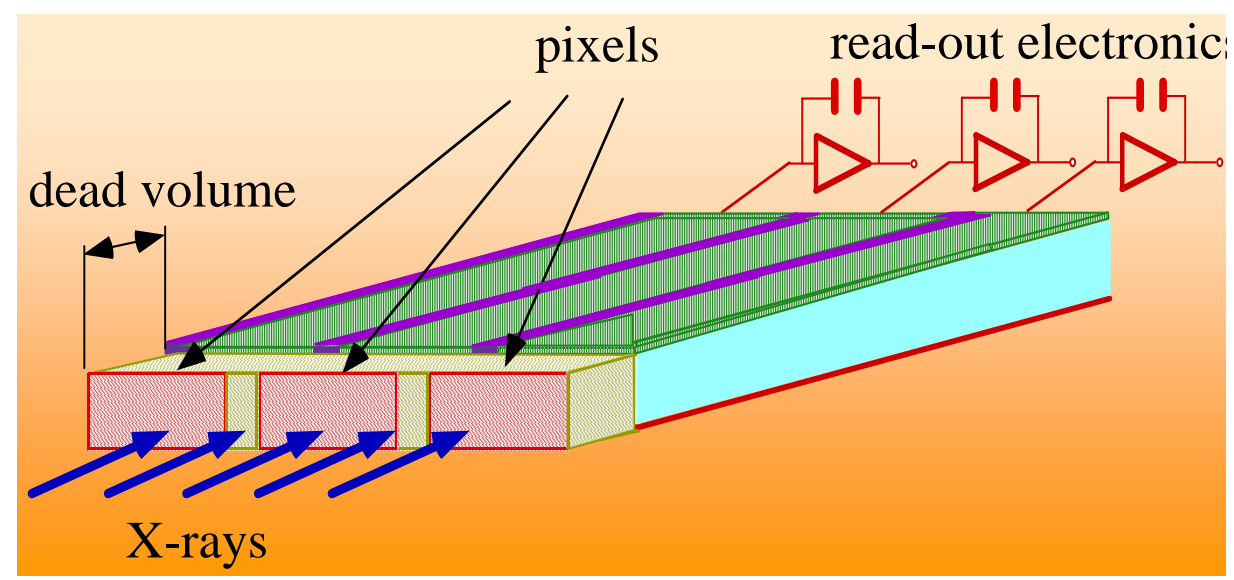

Fig. 1



Fig. 2



Fig. 3 


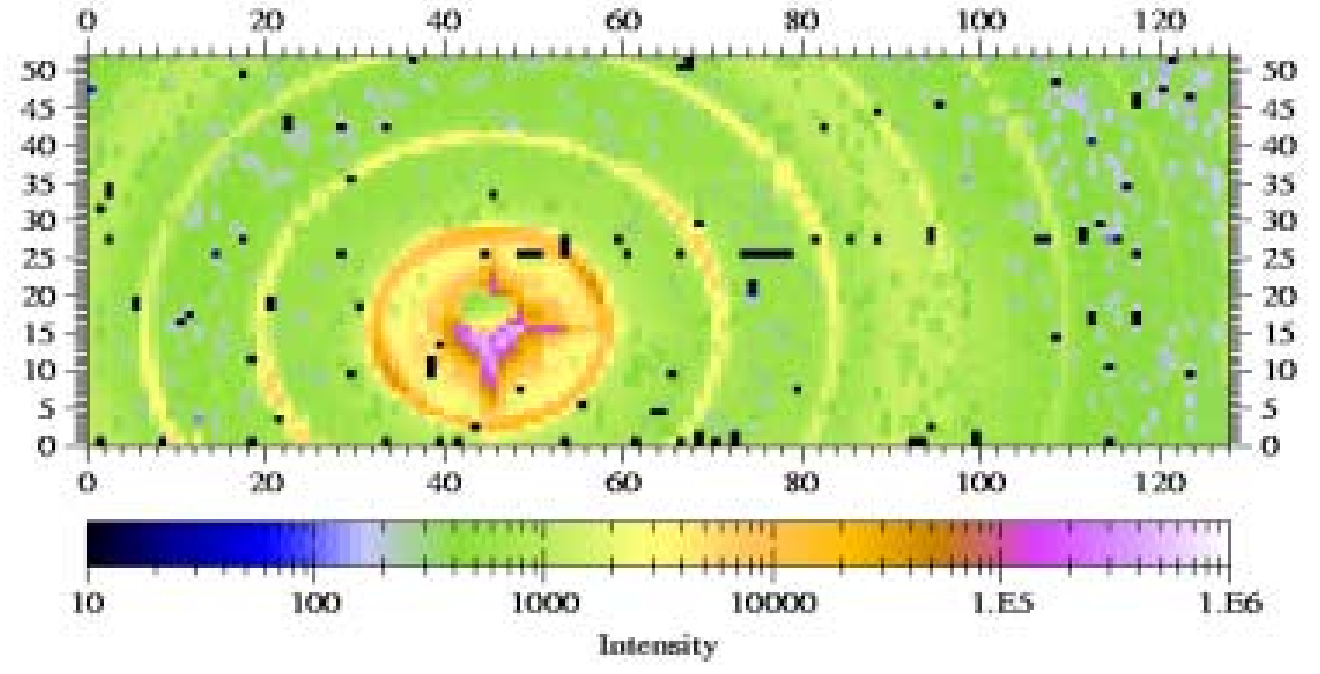

Fig. 4

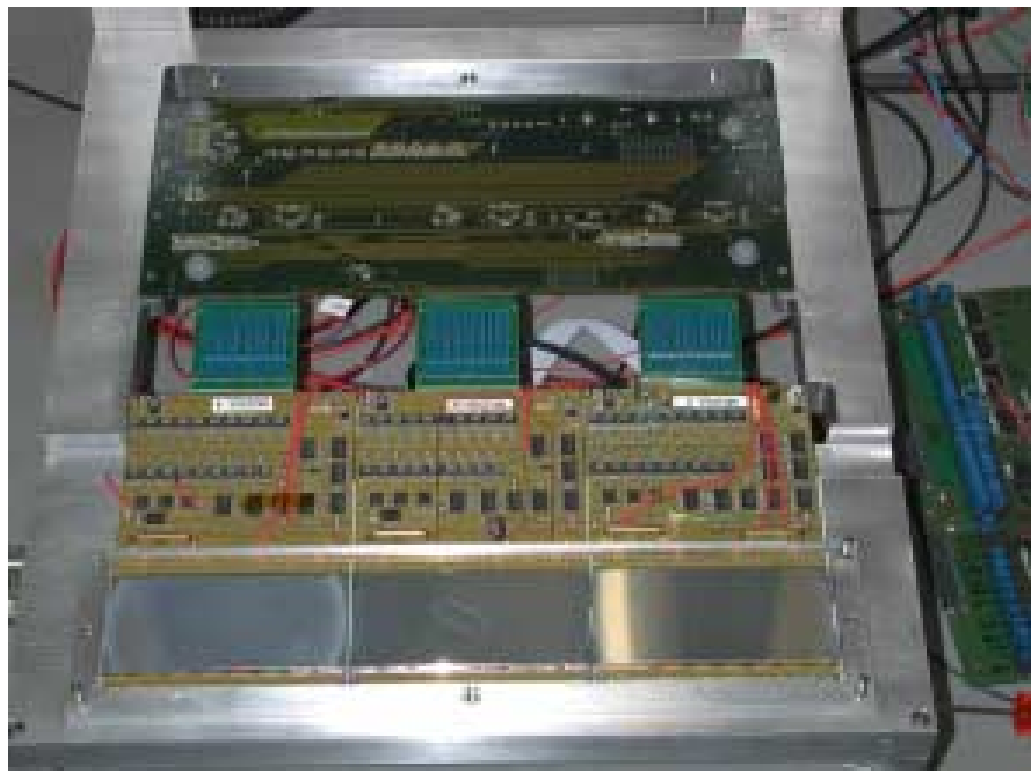

Fig. 5

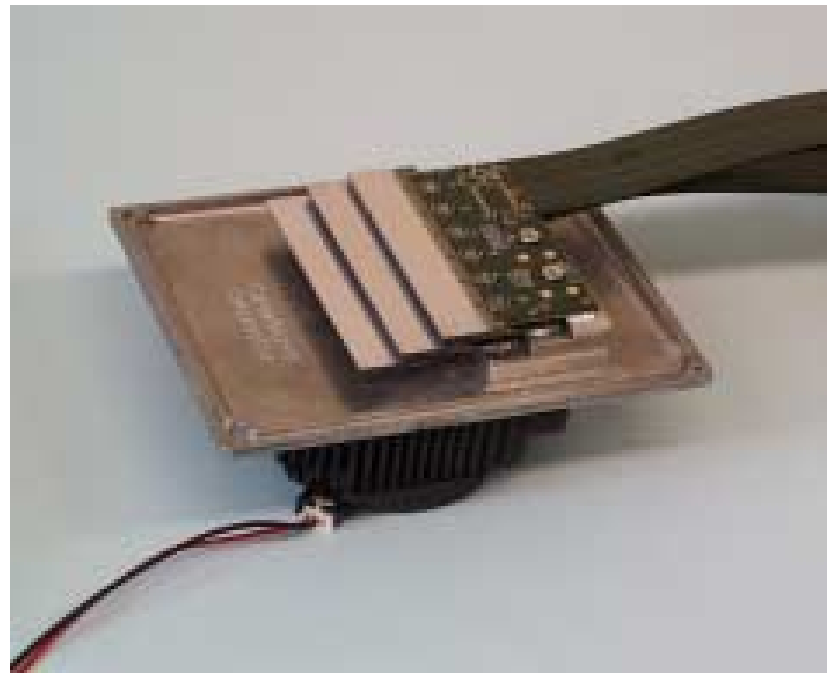

Fig. 6 


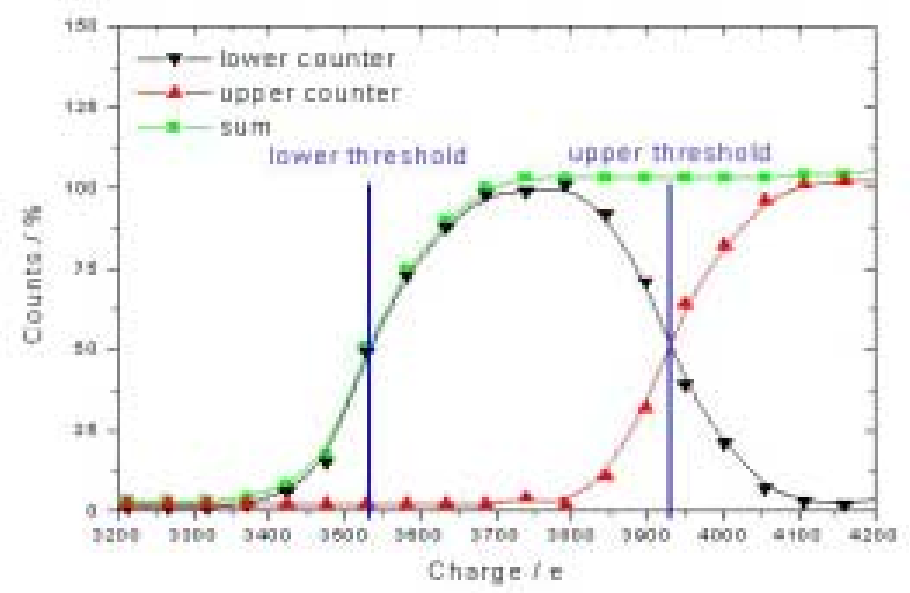

Fig. 7

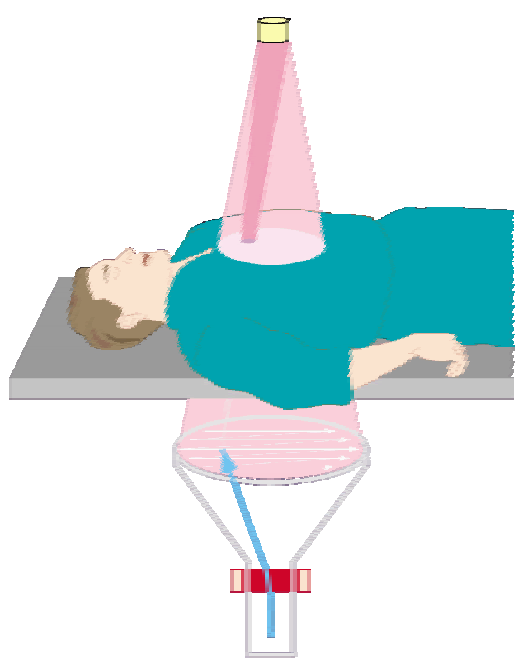

Fig. 8 


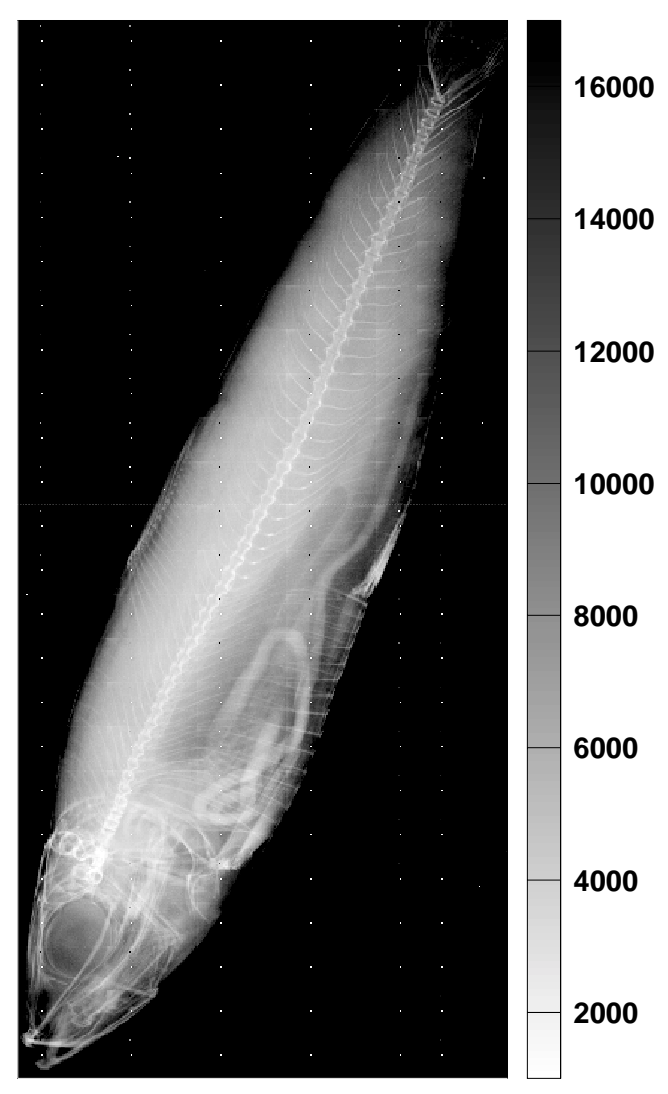

Fig. 9

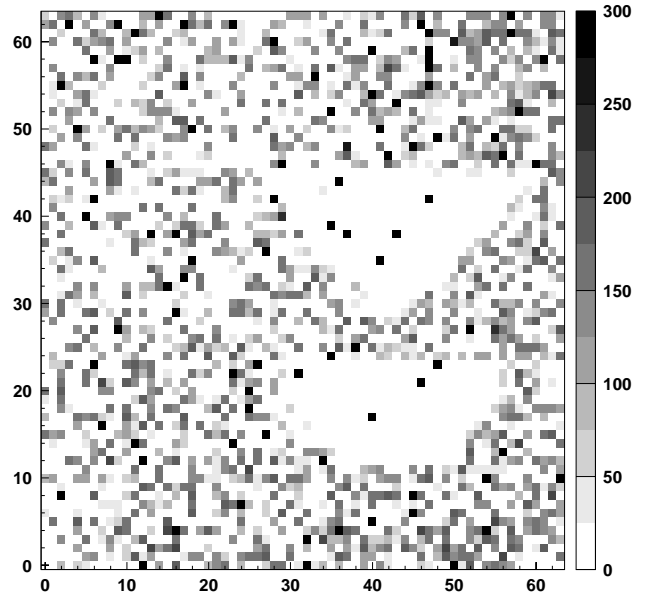

Fig. 10(a)

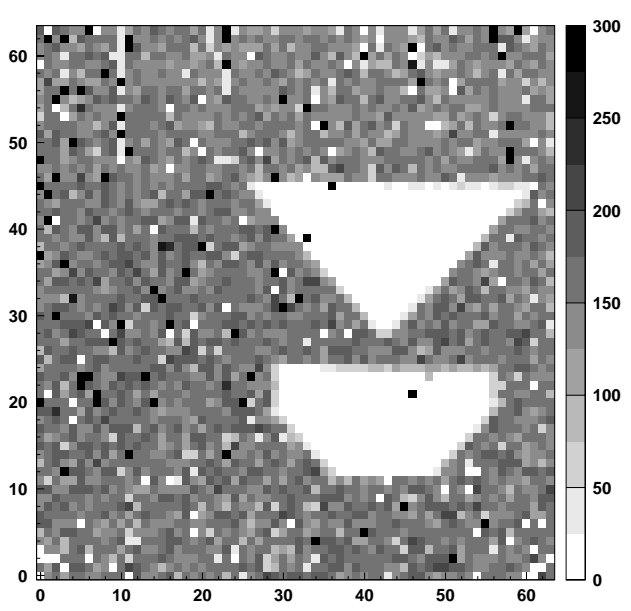

Fig. 10(b) 


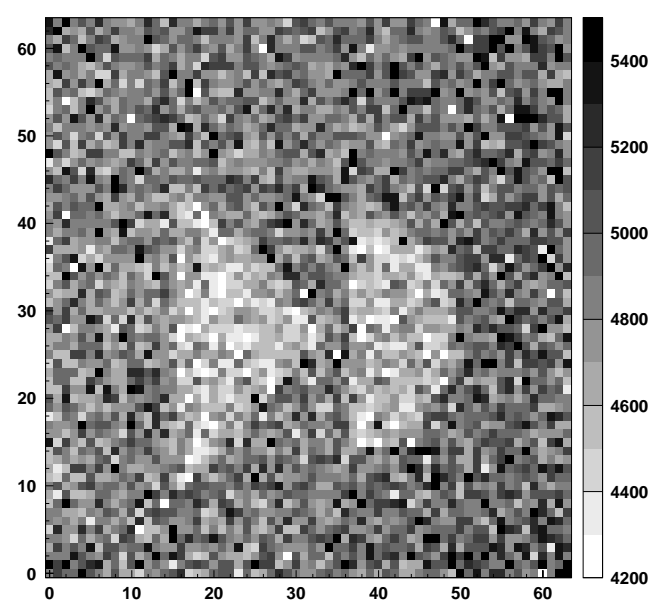

Fig. 11(a)



Fig. 12(a)

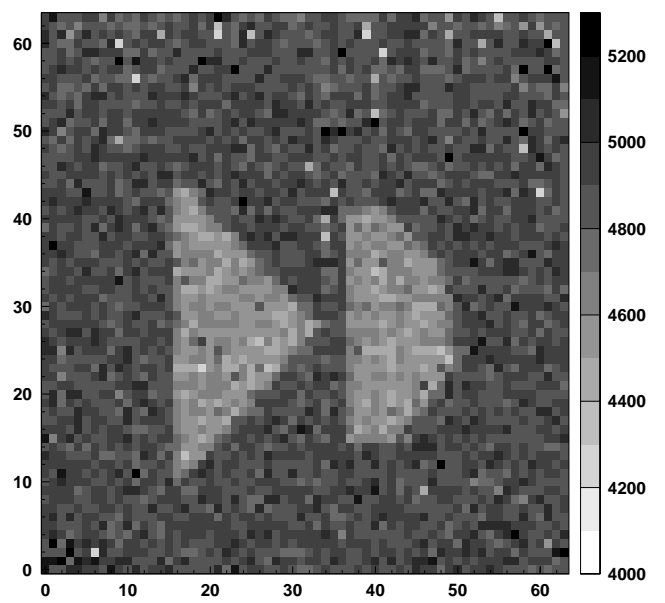

Fig. 13(a)

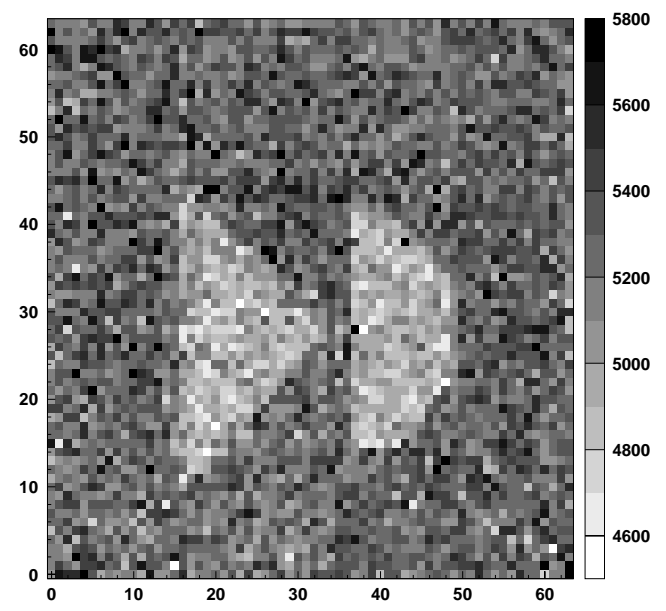

Fig. 11(b)

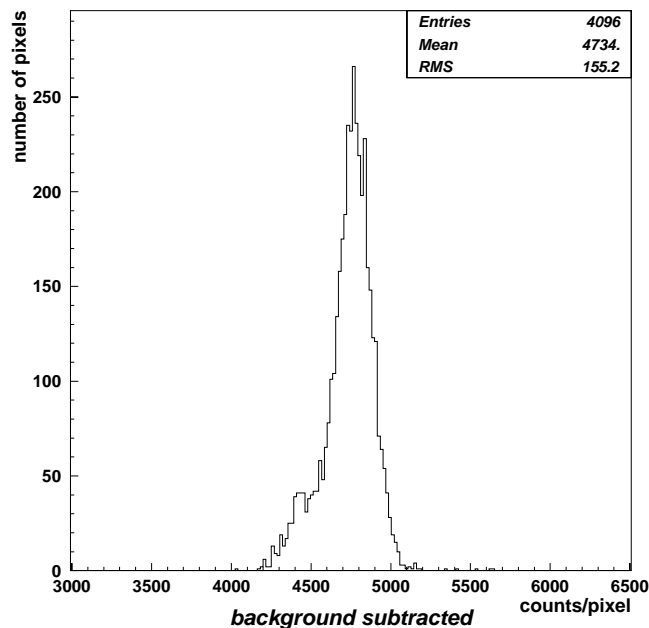

Fig. 12(b)

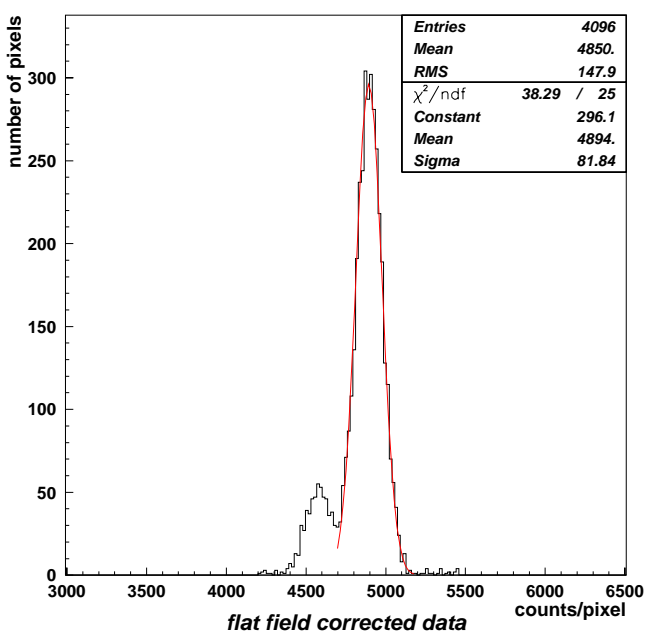

Fig. 13(b) 


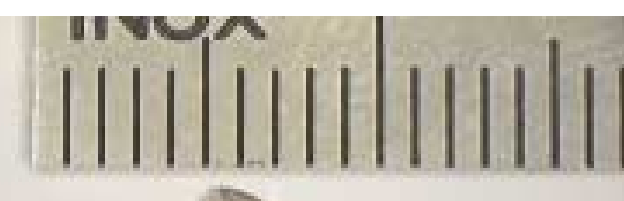

Fig. 14

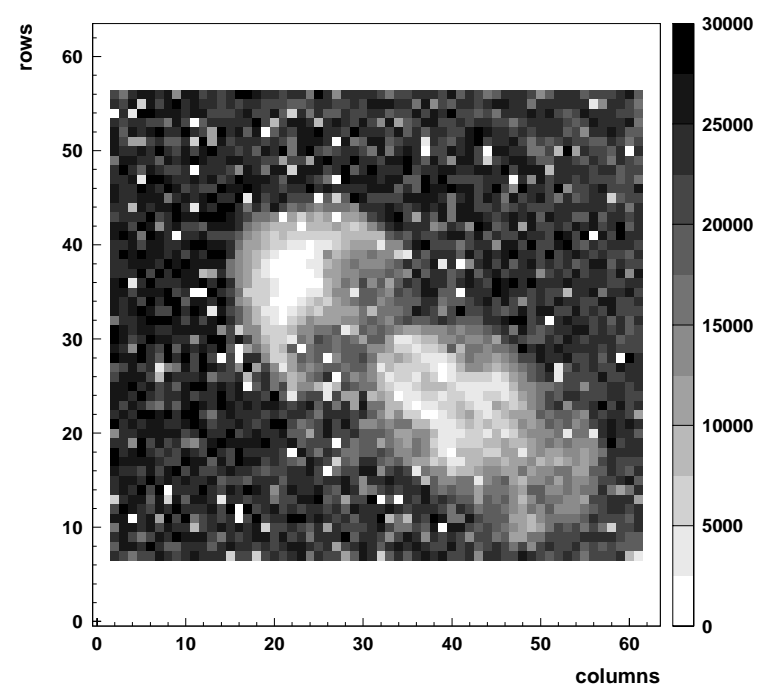

Fig. 15(a)

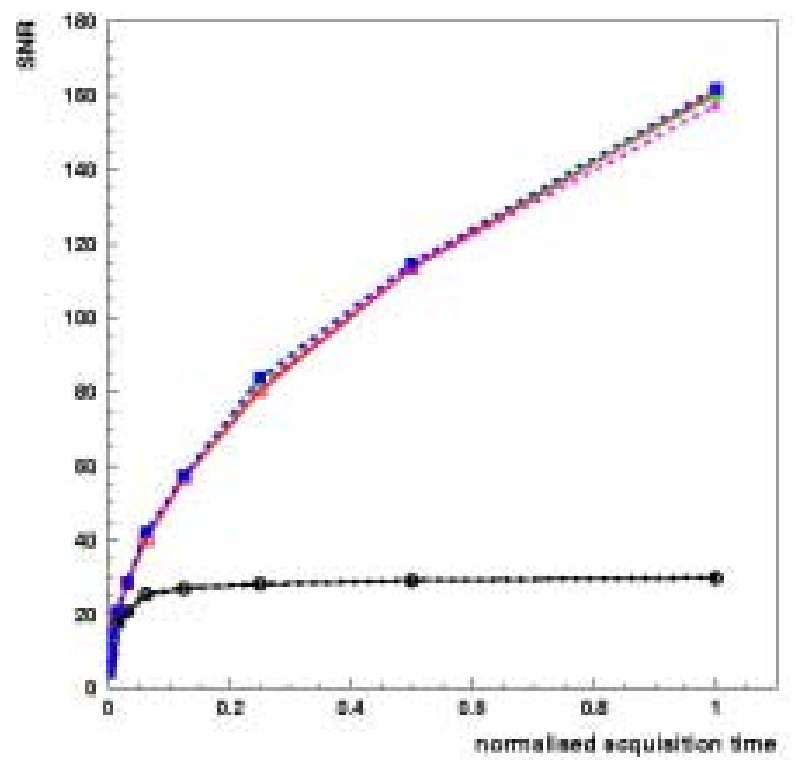

Fig. 16

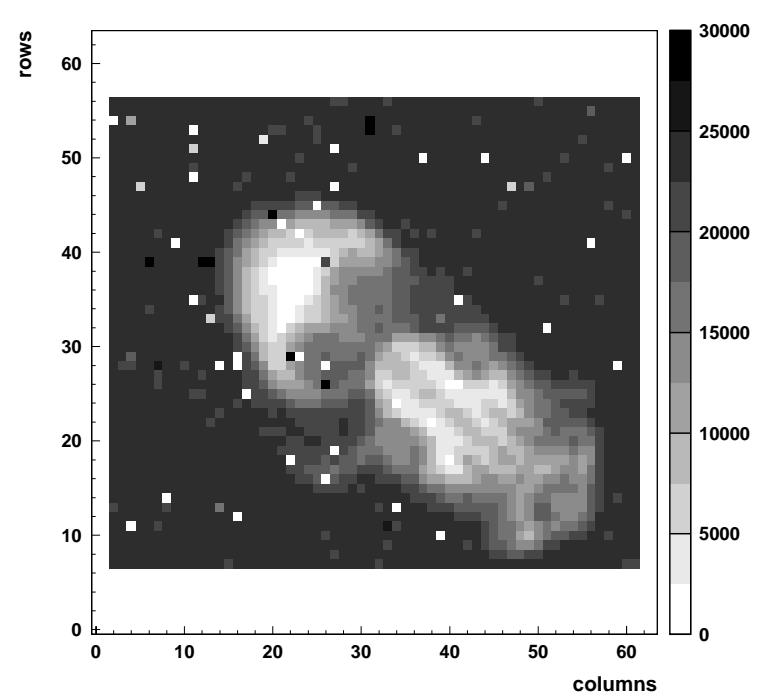

Fig. 15(b)

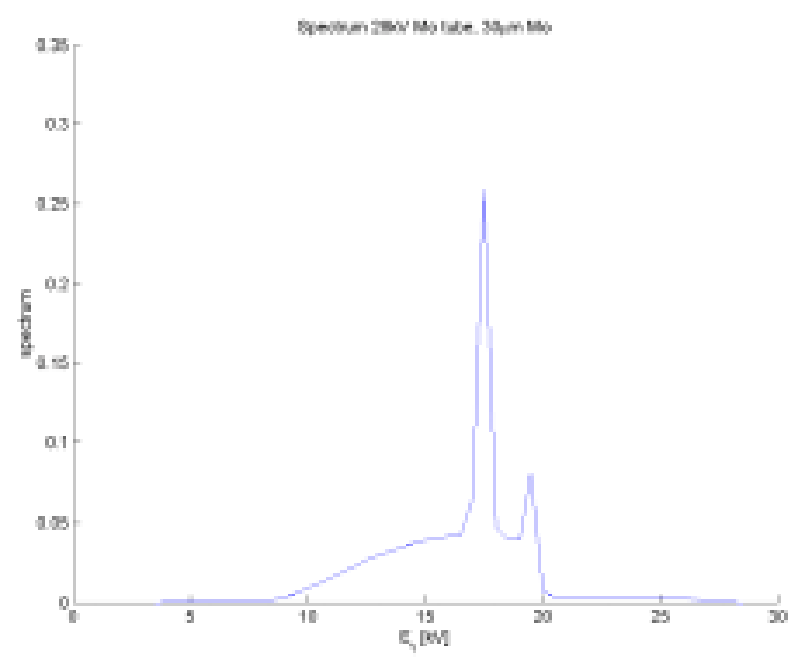

Fig. 17 




Fig. 18
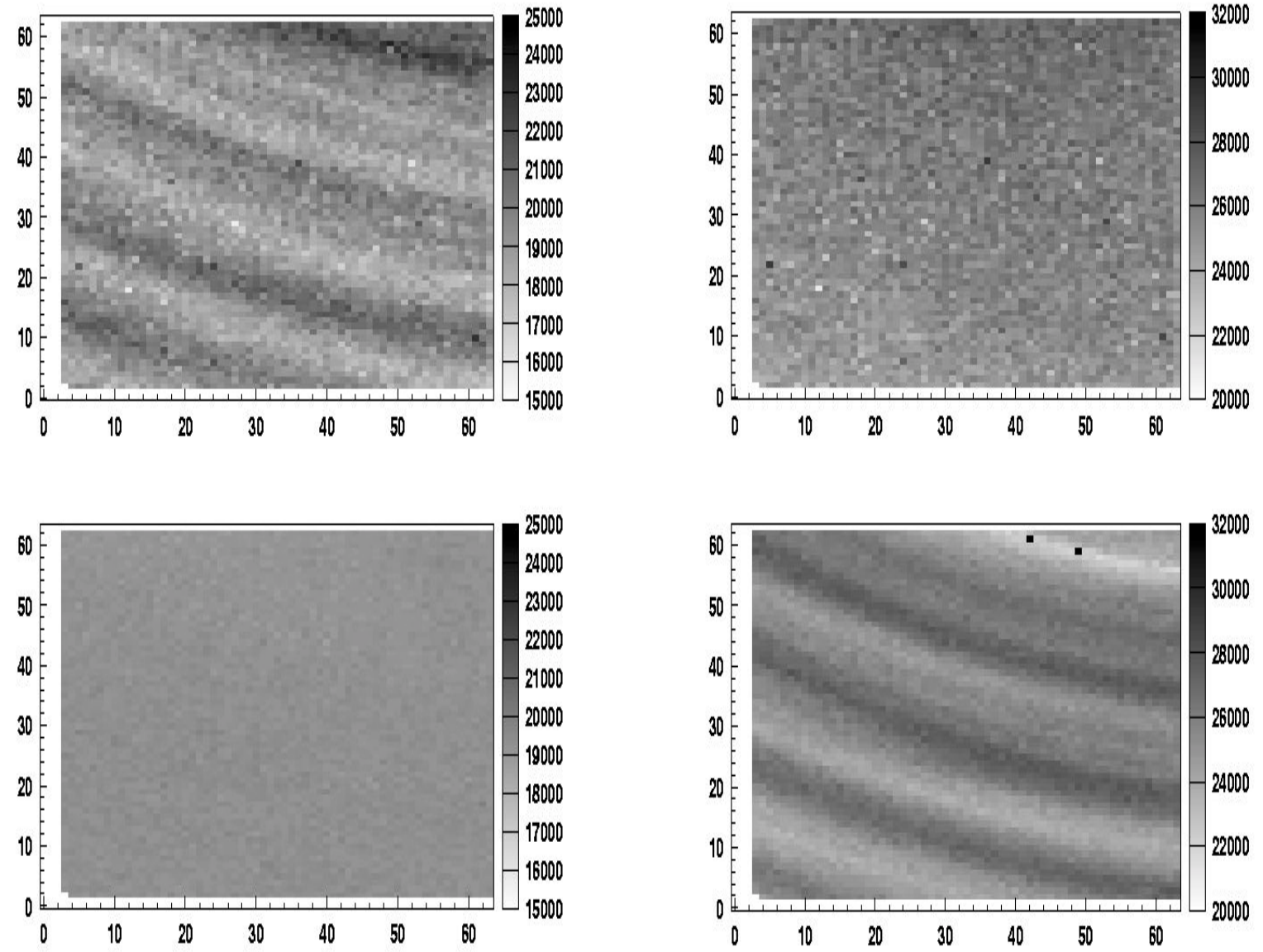

Fig. 19(a)

Fig. 19(b) 




Fig. 20

Fig. 21
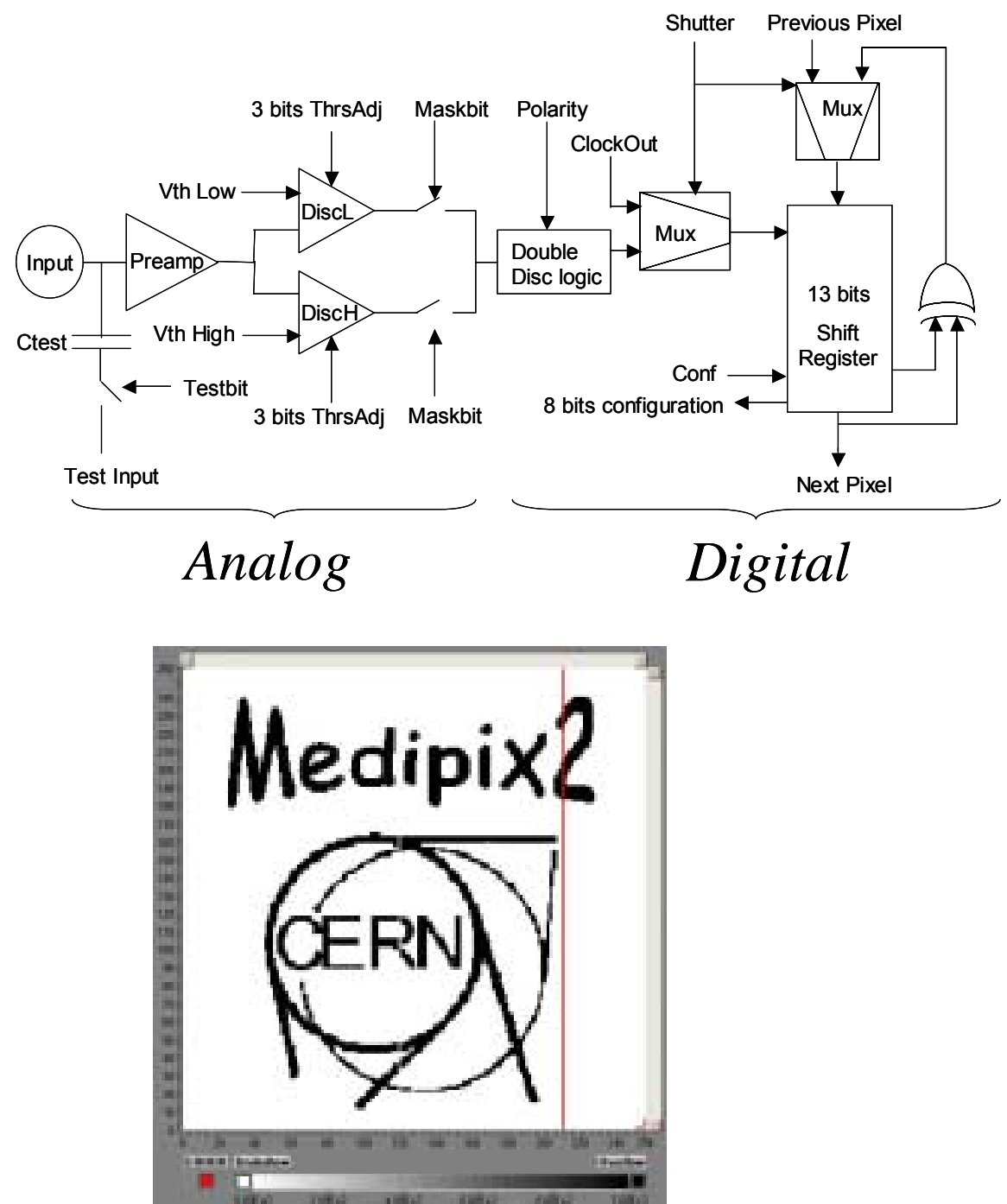

Fig. 22 
Fig. 1: Illustration of the edge-on principle: the X-rays impinge parallel to the sensor strips which results in a sort of pixel geometry with the pixel dimensions given by the strip pitch in one direction, the sensor thickness in the other one and the strip length defining roughly the absorption depth. There is a dead layer at the entrance side of the radiation that has to be minimised.

Fig. 2: Illumination of a powder sample with monochromatic X-rays produces diffraction cones whose characteristic aperture contains the information about the atomic composition of the sample.

Fig. 3: Pixel module including ten XPAD chips (rear side of the chips is visible) bump-bonded to a silicon sensor (underneath the chips).

Fig. 4: Diffraction image of a silver behenate sample showing 7 orders of diffraction rings taken with a $20 \mathrm{keV}$ X-ray beam and a XPAD module.

Fig. 5: Photograph of a PILATUS detector consisting of 3 PILATUS modules. The active area corresponds to $24 \times 3.5 \mathrm{~cm}^{2}$ and is read out by 48 SLS chips.

Fig. 6: Illustration of the 'roof tile' architecture to avoid dead space between detector modules.

Fig. 7: Working principle of the window logic: the counter corresponding to the low threshold (set at $\sim 3530 \mathrm{e}^{-}$) registers all the events in-between the upper and lower threshold. As soon as the signal passes the upper threshold (at $\sim 3930 \mathrm{e}^{-}$) the lower counter is turned off and the events are stored in the upper counter. The total response is $100 \%$.

Fig. 8: In the scanning-beam digital x-ray system an electron beam scans a large transmission target. The X-rays pass though a collimator with $100 \times 100$ aperture holes and get focused onto the CdZnTe photon counting pixel array.

Fig. 9: X-ray image of a sardine taken with a Mo X-ray tube $+30 \mu \mathrm{m}$ Mo filter, $25 \mathrm{kV}, 10 \mathrm{mAs}$, distance target-object $\sim 50 \mathrm{~cm}$. A Medipix1 Si detector was stepped for one detector width in $\mathrm{x}$ and half a detector width in $\mathrm{y}$; the acquisition time per image was $500 \mathrm{~ms}$. No image correction was applied. The thickness of the fish bones corresponds roughly to the pixel size.

Fig. 10: Two thin silicon objects imaged with a Medipix1 Si detector and a ${ }^{55} \mathrm{Fe}$ source. The grey levels correspond to different numbers of counts in each pixel. For image (a) no threshold adjustment was applied in contrary to image (b), where the pixel-wise 3-bit threshold tuning facility was used.

Fig. 11: Image of two low-contrast objects taken with a ${ }^{109} \mathrm{Cd}$ souce and a Medipix1 Si detector (fig. (a)). Fig. (b) was made under the same conditions except that the thresholds in each pixel were tuned.

Fig. 12: Image of two low-contrast objects taken with a ${ }^{109} \mathrm{Cd}$ souce and a Medipix1 Si detector and subtracting a flood image (fig. (a)) and its 1-dimensional representation (fig. (b)). 
Fig. 13: Image of two low-contrast objects taken with a ${ }^{109} \mathrm{Cd}$ souce and a Medipix1 Si detector and applying a flat field correction (fig. (a)); the 1-dimensional representation is shown in (fig. (b)).

Fig. 14: Photograph of the fly used to produce images $14(\mathrm{a})$ and (b).

Fig. 15: Image of the fly from fig. 14 taken with a Medipix1 Si detector and a ${ }^{55} \mathrm{Fe}$ source. Threshold adjustment was applied for image (a). Adding a flat field correction improves the image quality significantly (b).

Fig. 16: SNR as a function of acquisition time (covering the full 15-bit dynamic range of the Medipix1 chip). The dashed-dotted curve represents the SNR measured with flood fields and a Mo tube, $30 \mu \mathrm{m}$ Mo filter, $28 \mathrm{kV}, 2 \mathrm{~mA}$. Using only the threshold adjustment facility, a SNR of $\sim 30$ is reached (global threshold of the chip corresponded to $\sim 8.5 \mathrm{keV}$ ). Applying a flat field correction the SNR of the Medipix1 Si detector follows perfectly the theoretical Poisson limit indicated by the solid line and open squares.

Fig. 17: Normalised spectrum of a Mo tube at $28 \mathrm{kV}$ with a $30 \mu \mathrm{m}$ Mo filter.

Fig. 18: SNR as a function of normalised acquisition time. The same measurement setup was used as for fig. 16, except that the detector bias voltage was reduced to $17 \mathrm{~V}$ (under-depletion).

Fig. 19: Fig. 19(a) (top) shows bands of different charge collection efficiency in a flood image from an under-depleted Medipix1 Si detector, which disappear applying a flat field correction (bottom). A flood image of the same detector biased at $80 \mathrm{~V}$ is shown in fig. (b) (top). Multiplying with the wrong flat field coefficients from the under-depleted case the inverse band structure appears (bottom), but the local pixel-to-pixel inhomogeneities still are suppressed. This points to a fixed pattern noise in the silicon sensor.

Fig. 20: Standard deviation of flood images normalised to the Poisson limit as a function of Perspex layer thickness [cm]. Different dashed curves correspond to different flat field corrections (see text for explanation). The results indicated with filled circles are without any flat field correction.

Fig. 21: Schematic block diagram of the Medipix2 pixel cell.

Fig. 22: Image generated in a Medipix2 chip by loading a mask and pulsing the whole pixel matrix at once with 1000 pulses. Two bad columns can be observed. 